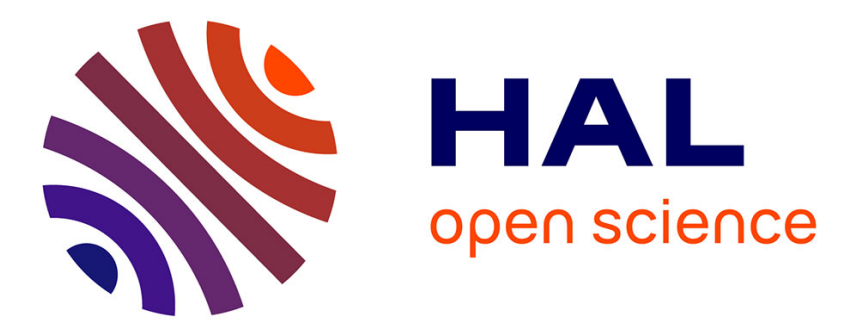

\title{
Optimization of a Cruciform Specimen Geometry for the Identification of Constitutive Parameters Based Upon Full-Field Measurements
}

Morgan Bertin, François Hild, Stéphane Roux

\section{- To cite this version:}

Morgan Bertin, François Hild, Stéphane Roux. Optimization of a Cruciform Specimen Geometry for the Identification of Constitutive Parameters Based Upon Full-Field Measurements. Strain, 2016, 52, pp.307 - 323. 10.1111/str.12178 . hal-01383975

\section{HAL Id: hal-01383975 \\ https://hal.science/hal-01383975}

Submitted on 19 Oct 2016

HAL is a multi-disciplinary open access archive for the deposit and dissemination of scientific research documents, whether they are published or not. The documents may come from teaching and research institutions in France or abroad, or from public or private research centers.
L'archive ouverte pluridisciplinaire HAL, est destinée au dépôt et à la diffusion de documents scientifiques de niveau recherche, publiés ou non, émanant des établissements d'enseignement et de recherche français ou étrangers, des laboratoires publics ou privés. 


\title{
Optimization of a cruciform specimen geometry for the identification of constitutive parameters based upon full-field measurements
}

\author{
Morgan B. R. Bertin*, François Hild and Stéphane Roux \\ LMT (ENS Cachan, CNRS, Université Paris-Saclay) \\ 61 avenue du Président Wilson, 94235 Cachan, France
}

\begin{abstract}
A methodology is proposed to optimize a specimen shape in a biaxial testing machine for the identification of constitutive laws based on full-field measurements. Within the framework of Finite Element Model Updating (FEMU) and Integrated Digital Image Correlation (IDIC), the covariance matrix of the identified material parameters due to acquisition noise is computed and its minimization is the basis of the proposed shape optimization. Two models are investigated; first, a linear elastic law, and second, an elastoplastic law with linear kinematic hardening. Two optimal fillet radii sets are assessed for the two investigated laws based on the minimization of the identification uncertainty.

Keywords: Digital Image Correlation (DIC), Integrated Digital Image

Correlation (IDIC), Finite Element Model Updating (FEMU), Full-field measurements, Identification, Optimization
\end{abstract}

\footnotetext{
* Corresponding author

Email address: \{morgan.bertin; francois.hild; stephane.roux\}@lmt.ens-cachan.fr (Morgan B. R. Bertin*, François Hild and Stéphane Roux)
}

Preprint submitted to Journal of Strain 


\section{Introduction}

One of the main issues of mechanical engineering is to understand, describe and predict how materials behave and fail. For many industrial applications, the optimization with respect to mass for instance pushes toward accurate and hence complex constitutive laws. Just to mention a very early example, elastoplastic flow in sheet metal forming prompted Hill [1] to emphasize on the need for properly describing anisotropy to account for the influence of the orientation of the sheet with respect to the rolling direction. Together with the complexity of constitutive laws comes a rapid increase in the number of material parameters to be identified in order that the model matches the load / displacement relationship as observed in either one or several mechanical test(s). This inflation of parameters implies an increased difficulty to measure them in well-suited experiments. In particular, plastic anisotropy calls for multiaxial testing.

Due to the complexity of designing multiaxial testing devices, one compromise was to consider flat cruciform specimens [2. The authors showed the benefit provided by such samples for biaxial fatigue studies with numerical simulations. However, only theoretical and modeling approaches were performed [3]. Kelly 4] carried out "virtual experiments" to characterize materials with multiaxial loadings. Finally, the first in-plane biaxial loading system has been proposed by Makinde et al. [5, 6, 7]. The authors proposed an original experimental device to prescribe biaxial tension in the plane of a cruciform specimen to study plasticity. Afterward, ad hoc systems were proposed to perform biaxial tension [8, 9, 10, using uniaxial testing machines [1]. 
Other authors [6, 12, 13, 14, 11] studied plasticity under biaxial loading and applications to constitutive modeling [15]. Eftis et al. [16] studied crack propagation under biaxial loadings. Kuwabara [8] prescribed different loading paths with both cruciform specimens and bending tests. It appears that the most flexible and versatile experimental system is related to in-plane biaxial tests of cruciform specimens [7, 14, 17] among different systems [18, 12, 17. Some of them involve complex loading conditions [19, 20, 21, 22, 23].

Two main issues raised by the above-cited authors were i) How to reveal specific material behaviors? and ii) How to quantify and identify material behaviors? The first issue deals with the design of experimental systems. The second one is related to measurement and identification procedures. These two aspects (i.e., measurement and identification) are linked. For example, in the case of the identification of constitutive parameters, the main guide to the design of cruciform specimens, namely homogeneity, was motivated by the measurement methods used (e.g., strain gauges or extensometers).

Even if innovative sensors were proposed [24], the total number of sensors remained fairly small. This constraint has led to specific optimization criteria 25. They accounted for three aspects, namely, the homogeneity of the zone over which the phenomena occur and the level of strains and stresses in order to have good measurements with strain gauges [12, 8, 26, 14]. The homogeneity requirement led for instance to specific features such as slits to cancel out Poisson's effect occurring on specimen arms and yielding larger homogeneous zones [25, 27, 28, a thinner central zone to concentrate the strains [19, 29] and 
a minimum number of discontinuities [13, 30] were also considered.

However, the stress and strain uniformity is not necessarily required when identification techniques accounting for heterogeneous fields are considered. First introduced by Kavanagh and Clouth [31] the so-called "Finite Element Model Updating" (FEMU) technique aims to identify the constitutive parameters while updating the numerical model to the experimental data. Lecompte et al. [13] proposed a mixed numerical-experimental technique to identify orthotropic parameters of metals based on full-field measurements. With a biaxial tensile test the FEMU technique was used to compare measured and computed strain fields (i.e., FEMU- $\epsilon$ ). This method is one out of several procedures based on full-field measurements [32, 33. Similarly, Schmaltz et al. 34] made use of stereo-DIC measurements and FEMU-U (i.e., comparison between measured and computed displacement fields) to identify plastic laws for four different cross-shaped geometries. The key point here was the introduction of full-field measurements, that is, a considerable increase in the density of measurement points. This abrupt transition from sparse to dense measurements is a major change of perspective.

FEMU can be extended to Integrated Digital Image Correlation (IDIC [35, 36. 37.) to couple measurement and identification procedures. Unlike other identification techniques such as FEMU, IDIC relies directly on the images to determine material parameters. It allows standard finite element codes to be used in a non-intrusive way. Further, when properly weighted it can be shown that for small noise levels weighted FEMU and IDIC lead to similar covariance 
matrices of the sought parameters [38].

The fact that full-field measurements are performed will lead to new approaches to mechanical test design. The geometry and load history will be chosen to optimize the sensitivity of the test with respect to the sought constitutive parameters. The present paper aims to pave the way to a systematic optimization of test design tailored for an anticipated constitutive law. Here only the specimen geometry is optimized for the sake of simplicity and the loading path is considered as fixed. The target is formulated in terms of the quality of the measurement of constitutive parameters (i.e., the least uncertainty is sought). After presenting the theoretical background, a single parameter optimization will be performed, based on the radius of a cruciform specimen fillet. An artificial (i.e., numerical) case will be studied where the uncertainty is computed from an experimentally representative noise level in the entire data acquisition and treatment chain. Linear elasticity and elastoplasticity (with linear kinematic hardening) will be discussed to highlight the key role played by the formulation of the sought objective, and henceforth the constitutive law parameterization. The formalism proposed herein is transparent to more complex constitutive (e.g., including various hardening postulates). To illustrate this test case, constitutive parameters are chosen as representative of $17-7$ PH (Precipitation-Hardened) stainless steel [39, 37], which is known for its very good fatigue properties [40, 41]. Let us stress however that the present optimization is based on noise uncertainty and omits possible bias due to model error, which can hardly be addressed a priori (or very artificially). 


\section{Optimization strategy}

To optimize the geometry of cross-shaped samples, Schmaltz et al. [34] propose to use so-called stress-stress and major-minor strain graphs. The first ones are determined via numerical simulations, whereas the second ones can be assessed experimentally via full-field measurement techniques. The present optimization is based on the covariance matrix of the identified material parameters, which indicates the identification uncertainty associated with a chosen constitutive law. The identifiability of constitutive parameters is estimated using the quantification of their influences on experimentally observable (i.e., measured) quantities (e.g., displacement or temperature fields, resultant forces). Their influences are expressed with quantities called sensitivity fields [42, 13, i.e., gradients of the observables with respect to the to-be-identified parameters. Considering one parameter variation and identical boundary conditions, there exists one sensitivity field for each material parameter. The latter ones are collected in a vector $\{\boldsymbol{p}\}$ whose components are conveniently designed to be dimensionless. This can always be performed through a normalization with the expectation values of the parameters (i.e., nominal values). For convenience, it is possible to define as constitutive parameters logarithms of moduli scaled by nominal values.

The sensitivity fields are to be computed numerically based on the current determination of the constitutive parameters as well as with boundary conditions that are obtained from DIC analyses. In the present study, the identification is based on full-field measurements and two related techniques, namely, 
FEMU and integrated DIC [38. Within those approaches, it is possible to track down the uncertainty from the measurement step down to the identification step. This uncertainty is collected into a global covariance matrix $\left[\boldsymbol{C}_{\boldsymbol{p}}\right]$

$$
\left[\boldsymbol{C}_{\boldsymbol{p}}\right]=\langle\{\delta \boldsymbol{p}\} \otimes\{\delta \boldsymbol{p}\}\rangle
$$

where the angular brackets $\langle\ldots\rangle$ denote the mathematical expectation of the enclosed argument. $\left[\boldsymbol{C}_{\boldsymbol{p}}\right]$ deals with all facets of the problem, namely, the geometry of the studied structure, the chosen constitutive law, the set of parameters, the boundary conditions, the measurement uncertainties and the identification method. This is important since it provides an estimate of the quality of the identified parameters through an uncertainty, and also because it gives a handle on how to optimize a mechanical test (in the present case through the shape of the specimen). The lower the value of this covariance, the more reliable the identification. The objective is to minimize the covariance matrix components with respect to the optimization parameters (here chosen to be associated with the specimen shape). However, because this covariance is a second order tensor, some more discussion is required on what is really meant by minimization, and this issue and some of its consequences will be discussed and illustrated below.

\subsection{Test and observables}

\subsubsection{Specimen geometry}

The testing machine is biaxial [37, which has the ability to prescribe an arbitrary tensile strain in two orthogonal directions, $\boldsymbol{e}_{\mathbf{1}}$ and $\boldsymbol{e}_{2}$, with opposite actuators that behave in a symmetric fashion so that the specimen center is 
motionless. The specimen shape is thus naturally cruciform (Figure 1). In the following, the four fillets are first assumed to have the same radius. The fillet radius will be the argument of the optimization problem. The region of interest is a square of area $30 \times 30 \mathrm{~mm}^{2}$. Figure 1 shows the triangular mesh used for the numerical simulation and the specimen geometry for a fillet radius equal to $r=2 \mathrm{~mm}$.

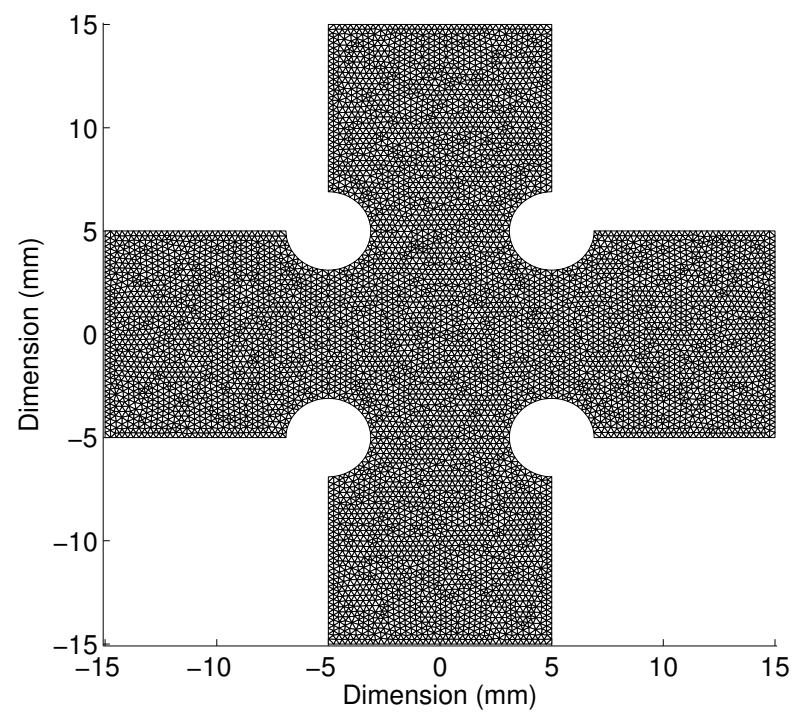

Figure 1: Mesh and geometry of the analyzed specimen with a fillet radius $r=2 \mathrm{~mm}$

This type of geometry was used to perform biaxial experiments on 17-7 PH stainless steel. It has been chosen regarding the manufacturing process and the machine capabilities, namely, $i$ ) Electrical Discharge Machining (EDM), ii) flat and thin biaxial specimens, $i i i$ ) the largest arm width available to ensure the 
largest observable surface, and $i v$ ) a sufficiently simple geometry with a small number of parameters to optimize. However, other optimization parameters could be investigated such as adding a hole to the specimen. It is worth noting that other geometries have been proposed, e.g., by Schmaltz et al. [34].

\subsubsection{Loading history}

As shown in Figure 2, a "nonproportional" loading path is prescribed. First a displacement along direction $\boldsymbol{e}_{1}$ is applied up to a maximum value $d_{1}=d^{*}$ while the transverse displacement is kept equal to $0(O A)$. Then, at fixed displacement $d_{1}$, the displacement $d_{2}$ is raised to reach an equibiaxial strain state, $d_{1}=d_{2}=d^{*}$ (point $B$ ) and finally both displacement amplitudes are reduced to zero at the same rate $(B O)$ so that in the displacement plane $\left(d_{1}, d_{2}\right)$ the loading path is a triangle.

The tested specimens are $0.3 \mathrm{~mm}$ thick [37. Thus, forces in both directions are chosen greater than or equal to zero to avoid buckling. The measured quantities are of two types. First, reaction forces in each arm are measured using load sensors. Second, at prescribed stages, images of the specimen surface are captured. They will be processed to measure displacement fields using DIC techniques discussed in details below. The simulated digital camera is able to acquire 16-bit gray scale images. The physical size of one pixel is $a=13.5 \mu \mathrm{m}$.

Each measurement is performed with a specific uncertainty. $\gamma_{F}^{2}$ denotes the variance of the force measurement, where all load cells are independent of each other. Similarly, images are subjected to a noise that will be considered to be Gaussian and white (i.e., uncorrelated). One key feature of the used DIC 


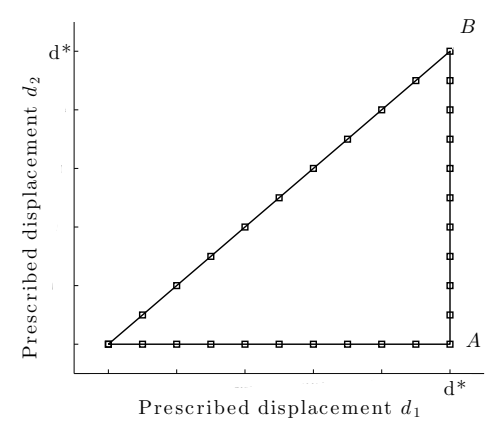

(a)

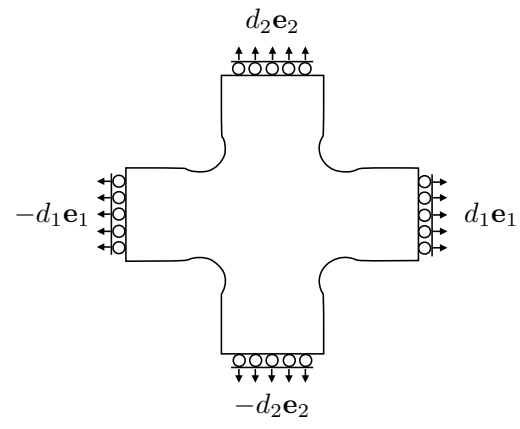

(b)

Figure 2: (a) Triangular loading history prescribed in the simulation. The squares depict the computed steps. (b) Boundary conditions prescribed on the sample in terms of longitudinal displacements. With the chosen loading history the center of the specimen is motionless

methodology is the fact that the effect of image noise on displacement uncertainty can be explicitly characterized. This is an extremely important feature as it allows for the design of the appropriate norm to be used in the FEMU identification procedure, and thereby to follow the impact of noise coming from either images or force sensors onto the uncertainty of the identified parameters 38. It is because of this complete chain that the optimization of the specimen shape can be addressed even when the specimen has not been tested yet.

\subsection{Covariance matrices}

The aim of this subsection is to introduce the general framework to estimate the covariance matrix $\left[\boldsymbol{C}_{\boldsymbol{p}}\right]$, which is the basis of the optimization procedure proposed herein. Two different routes will be followed. 


\subsubsection{Global DIC}

In the following, global DIC is considered. It relies on the registration of an image $f(\boldsymbol{x})$ in the reference configuration and a series of pictures $g(\boldsymbol{x}, t)$ in the deformed configurations indexed by time $t$. The gray level conservation is assumed

$$
f(\boldsymbol{x})=g(\boldsymbol{x}+\boldsymbol{u}(\boldsymbol{x}, t), t)
$$

where $\boldsymbol{u}(\boldsymbol{x}, t)$ is the sought displacement field. The problem consists of minimizing the norm of the gray level differences over the whole Region of Interest (ROI)

$$
\begin{aligned}
\chi_{o f}^{2} & =\|g(\boldsymbol{x}+\boldsymbol{u}(\boldsymbol{x}, t), t)-f(\boldsymbol{x})\|_{C_{n}}^{2} \\
& \equiv \sum_{\Omega} \sum_{\Omega}(g(\boldsymbol{x}+\boldsymbol{u}(\boldsymbol{x}, t), t)-f(\boldsymbol{x}))\left[\boldsymbol{C}_{\boldsymbol{n}}(\boldsymbol{x}, \boldsymbol{\xi})\right]^{-1}(g(\boldsymbol{\xi}+\boldsymbol{u}(\boldsymbol{\xi}, t), t)-f(\boldsymbol{\xi}))
\end{aligned}
$$

where $\left[\boldsymbol{C}_{\boldsymbol{n}}(\boldsymbol{x}, \boldsymbol{\xi})\right]$ denotes the covariance matrix of gray level noise for pixels $\boldsymbol{x}$ and $\boldsymbol{\xi}$. In this expression $\Omega$ denotes the ROI. Although seldom used, it can be shown that this norm is the best suited one. At convergence the residual $(g(\boldsymbol{x}+\boldsymbol{u}(\boldsymbol{x}, t), t)-f(\boldsymbol{x}))$ should be a statistically representative sampling of the noise for which the covariance has been computed.

Assuming that acquisition noise is Gaussian and white induces that the covariance matrix $\left[\boldsymbol{C}_{\boldsymbol{n}}(\boldsymbol{x}, \boldsymbol{\xi})\right]$ is equal to $\gamma_{f}^{2}[\delta(\boldsymbol{x}, \boldsymbol{\xi})]$, where $[\delta(\boldsymbol{x}, \boldsymbol{\xi})]$ is the Kronecker delta matrix, and $\gamma_{f}$ the standard deviation (expressed in gray levels) of noise. Equation 2.4 then reduces to the sum of squared differences between the deformed image corrected by the measured displacement $\boldsymbol{u}(\boldsymbol{x}, t)$ and the 
reference image (written for each time $t$ independently)

$$
\chi_{f}^{2}(t)=\frac{1}{2 \gamma_{f}^{2}|\Omega|} \sum_{\Omega}\left((g(\boldsymbol{x}+\boldsymbol{u}(\boldsymbol{x}, t), t)-f(\boldsymbol{x}))^{2}\right.
$$

that is minimized with respect to the sought displacement fields $\boldsymbol{u}(\boldsymbol{x}, t) .|\Omega|$ denotes the area in terms of the number of pixels the ROI contains. Noise is assumed to affect each image independently (including the reference one, which is responsible for the factor of $1 / 2$ coming as a multiplicative term in this functional).

In the following, the noise amplitude $\gamma_{f}$ will be considered as constant in space and time, and independent of gray level so that it could have been dropped from the expression of the functional $\chi_{f}^{2}(t)$ without consequences. However, it is introduced here [43, 38, so that at optimal registration, acquisition noise will be responsible for an expectation value of this functional equal to unity. A similar strategy will be followed in the sequel. Let us note that although time (indexing an image series) is an important aspect of identification because it is crucial to access the different stages of loading that will be sensitive to different features of the constitutive law, this section presents tools that are relative to image pairs. Treating a complete image series will simply consist of summing the different contributions of image pairs. Thus, the time parameter $t$ is dropped for the remainder of this section to simplify the notations.

The displacement field is decomposed onto a basis of functions $\boldsymbol{\psi}_{n}(\boldsymbol{x})$ that is selected at will

$$
\boldsymbol{u}(\boldsymbol{x})=\sum_{n} u_{n} \boldsymbol{\psi}_{n}(\boldsymbol{x})
$$


where $\boldsymbol{\psi}_{x}$ are shape functions in the present case, and $u_{n}$ the unknown degrees of freedom. Ideally, the number of these fields should be kept as small as possible (to reduce uncertainty on their amplitude) yet large enough to capture the anticipated variety or heterogeneity of the actual experimental field. The minimization of $\chi_{f}^{2}$ is achieved by successive linearizations and corrections, using Newton-Raphson's scheme

$$
[\boldsymbol{M}]\{\delta \boldsymbol{u}\}=\left\{\boldsymbol{b}^{(i)}\right\}
$$

where $[\boldsymbol{M}]$ is the DIC matrix, $\{\delta \boldsymbol{u}\}$ the vector gathering all increments of measured displacement amplitudes, and $\left\{\boldsymbol{b}^{(i)}\right\}$ the residual column vector at iteration $i$. The DIC matrix reads

$$
M_{i j}=\sum_{\Omega}\left(\nabla f(\boldsymbol{x}) \cdot \boldsymbol{\psi}_{i}(\boldsymbol{x})\right)\left(\boldsymbol{\nabla} f(\boldsymbol{x}) \cdot \boldsymbol{\psi}_{j}(\boldsymbol{x})\right)
$$

and the right hand side term

$$
b_{i}=\sum_{\Omega}\left(\boldsymbol{\nabla} f(\boldsymbol{x}) \cdot \boldsymbol{\psi}_{i}(\boldsymbol{x})\right)(f(\boldsymbol{x})-g(\boldsymbol{x}+\tilde{\boldsymbol{u}}(\boldsymbol{x})))
$$

where $\tilde{\boldsymbol{u}}(\boldsymbol{x})$ is the current estimate of the displacement field.

The DIC matrix is of importance when evaluating the covariance matrix of the measured degrees of freedom due to image noise, $\left[\boldsymbol{C}_{\boldsymbol{U}}\right]=\langle\{\delta \boldsymbol{u}\} \otimes\{\delta \boldsymbol{u}\}\rangle$ [44, 43

$$
\left[\boldsymbol{C}_{\boldsymbol{U}}\right]=2 \gamma_{f}^{2}[\boldsymbol{M}]^{-1}
$$

When a specific random pattern is known then the above equations are to be used. In the present case, the design is to be done without a specific speckle 
pattern in hand. For this reason, it is useful to resort to a "mean-field" assumption for the estimation of the DIC matrix $[\boldsymbol{M}]$, and derived quantities such as the covariance matrix of the kinematic degrees of freedom $\left[\boldsymbol{C}_{\boldsymbol{U}}\right]$. This approximation is based on a scale separation of shape functions $\psi(x)$ and random pattern $f(\boldsymbol{x})$ and their statistical independence. If the expectation value of the gray level gradient in the reference image, $G_{f}^{2}=\left\langle(\nabla f)^{2}\right\rangle$, is introduced the mean-field approximation of $[\boldsymbol{M}]$ reads

$$
M_{i j} \approx \frac{G_{f}^{2}}{2} \sum_{\Omega} \boldsymbol{\psi}_{i}(\boldsymbol{x}) \cdot \boldsymbol{\psi}_{j}(\boldsymbol{x})
$$

Many choices of basis fields $\boldsymbol{\psi}_{n}$ are possible within the above framework of global DIC. Two specific choices will be considered hereafter. First, a finite element representation of the displacement 44. For instance, a triangular mesh with finite elements that are three-node triangles with linear displacement interpolation (T3) is the simplest and offers both flexibility for meshing and robustness for DIC. Second, FEMU, which is detailed in the next subsection, can be transported into the global DIC framework with basis functions that are computed numerically from parameter sensitivity fields [38]. This procedure, which is called integrated DIC (or IDIC), is detailed after the FEMU method.

The output of this first processing of images is i) a series of displacement fields (one for each acquired image past the reference one) as amplitudes $\{\boldsymbol{u}(t)\}$ of the chosen kinematic basis and ii) the complete covariance matrix $\left[\boldsymbol{C}_{\boldsymbol{U}}\right]$ due to noise. (Note that the latter is independent of time.) It will be the input of the following identification procedure. 


\subsubsection{Weighted FEMU}

FEMU is a classical identification strategy that is based on the minimization of the differences between measured quantities (i.e., forces and displacements where the latter ones are obtained from DIC analyses) on the one hand, and the corresponding quantities that are computed from a numerical simulation exploiting a constitutive model and boundary conditions of Dirichlet type extracted from the DIC analysis. One key feature needed to obtain the most reliable estimate is to weigh the used information according to its value. This weight is coming from the covariance matrix of the measurement.

Weighted displacement-based Finite Element Model Updating (FEMU-U) consists of computing the set of (dimensionless) constitutive parameters, $\{\boldsymbol{p}\}$, that minimizes $\chi_{U}^{2}$,45, 38

$$
\chi_{U}^{2}=\frac{1}{N_{U}}\left\{\boldsymbol{u}_{m}-\boldsymbol{u}_{c}\right\}^{t}\left[\boldsymbol{C}_{\boldsymbol{U}}\right]^{-1}\left\{\boldsymbol{u}_{m}-\boldsymbol{u}_{c}\right\}
$$

where $\chi_{U}^{2}$ is the weighted squared differences between measured, $\left\{\boldsymbol{u}_{m}\right\}$, and computed, $\left\{\boldsymbol{u}_{c}\right\}$, nodal displacements and $N_{U}$ the total number of kinematic degrees of freedom. Once again, the prefactor has been chosen so that, at convergence, noise in the measured displacement should, by itself, endow $\chi_{U}^{2}$ with a unitary value. Because the displacement field has generically a nonlinear behavior with respect to the constitutive parameters, an iterative scheme is implemented for the minimization of $\chi_{U}^{2}$ with respect to $\{\boldsymbol{p}\}$

$$
\{\delta \boldsymbol{p}\}^{(i+1)}=\left(\left(\left[\boldsymbol{S}_{U}\right]^{(i)}\right)^{t}\left[\boldsymbol{C}_{\boldsymbol{U}}\right]^{-1}\left[\boldsymbol{S}_{\boldsymbol{U}}\right]^{(i)}\right)^{-1}\left[\boldsymbol{S}_{U}\right]^{(i)}\left[\boldsymbol{C}_{\boldsymbol{U}}\right]^{-1}\left\{\boldsymbol{u}_{m}-\boldsymbol{u}_{c}^{(i)}\right\}
$$

where $\left[\boldsymbol{S}_{U}\right]^{(i)}$ is defined as the displacement sensitivity matrix with respect to 
material parameters $\{\boldsymbol{p}\}$

$$
\left[\boldsymbol{S}_{U}\right]^{(i)}=\frac{\partial\left\{\boldsymbol{u}_{c}^{(i)}\right\}}{\partial\{\boldsymbol{p}\}}
$$

that is expressed in pixels as the parameters $\{\boldsymbol{p}\}$ have been chosen dimensionless.

At convergence, the displacement sensitivity matrix does not depend any longer on the iteration number and hence, in the following, the index $(i)$ will be omitted whenever one does not refer to the practical numerical implementation where sensitivities have to be updated through iterations as the constitutive parameters are evolving. In particular, assuming that the noise level is small, the linearized relationship is used between the increments in $\{\boldsymbol{p}\}$ and the remaining discrepancy $\left\{\boldsymbol{u}_{m}-\boldsymbol{u}_{c}\right\}$ at convergence to interpret the displacement difference as the fluctuation in measured displacement induced by noise $\{\delta \boldsymbol{u}\}$. The resulting $\{\delta \boldsymbol{p}\}$ will be nothing but the fluctuating part of the identified parameters due to noise. The expectation value of $\left[\boldsymbol{C}_{\boldsymbol{p}}^{\boldsymbol{U}}\right]=\langle\delta \boldsymbol{p} \otimes \delta \boldsymbol{p}\rangle$ is the corresponding covariance matrix that contains the full characterization of the statistical variability (within the present framework of Gaussian noise and small perturbations).

Using the expression of the covariance of the displacement field from global DIC, Equation 2.12 at convergence becomes

$$
\{\delta \boldsymbol{p}\}=1 /\left(2 \gamma_{f}^{2}\right)\left[\boldsymbol{H}_{U}\right]^{-1}\left[\boldsymbol{S}_{U}\right][\boldsymbol{M}]\{\delta \boldsymbol{u}\}
$$

where the Hessian $\left[\boldsymbol{H}_{U}\right]$ is introduced

$$
\left[\boldsymbol{H}_{U}\right]=\left[\boldsymbol{S}_{U}\right]^{t}\left[\boldsymbol{C}_{\boldsymbol{U}}\right]^{-1}\left[\boldsymbol{S}_{U}\right]=1 /\left(2 \gamma_{f}^{2}\right)\left[\boldsymbol{S}_{U}\right]^{t}[\boldsymbol{M}]\left[\boldsymbol{S}_{U}\right]
$$

The covariance in the constitutive parameters due to the image noise is expressed 
as 38

$$
\left[\boldsymbol{C}_{p}^{U}\right]=\left[\boldsymbol{H}_{U}\right]^{-1}
$$

The reaction forces may also be measured and compared with the computed resultants, which themselves incorporate $i$ ) a constitutive law whose parameters will at convergence be adjusted to those of the material to be identified, and ii) displacement boundary conditions that are provided by DIC measurements on the boundary. Thus, the same approach is followed with the reaction forces for which $\chi_{F}^{2}$ is minimized

$$
\chi_{F}^{2}=\frac{1}{N_{F}}\left\{\boldsymbol{F}_{m}-\boldsymbol{F}_{c}\right\}^{t}\left[\boldsymbol{C}_{\boldsymbol{F}}\right]^{-1}\left\{\boldsymbol{F}_{m}-\boldsymbol{F}_{c}\right\}
$$

where $N_{F}$ is the number of load cells, $\left\{\boldsymbol{F}_{m}\right\}$ the measured forces, $\left\{\boldsymbol{F}_{c}\right\}$ the computed reaction forces with respect to the chosen material parameter set, $\left[\boldsymbol{C}_{\boldsymbol{F}}\right]$ the covariance matrix of the measured loads (in the present case it is assumed that the load measurements are uncorrelated so that $\left[\boldsymbol{C}_{\boldsymbol{F}}\right]=\gamma_{F}^{2}[\boldsymbol{I}]$. However, it is assumed that the load uncertainty is proportional to the magnitude of the load, $\gamma_{F}^{2}=\rho_{1}^{2}|F|^{2}$. Moreover, it is easy at this stage to incorporate a minimum measurement uncertainty for the load cells by including an additional noise term whose variance $\rho_{0}^{2}$ is independent of the load level. This practically disqualifies all measurements of forces below $F_{\text {min }}=\rho_{0} / \rho_{1}$. Thus, it is assumed that [37]

$$
\gamma_{F}^{2}=\rho_{1}^{2}|F|^{2}+\rho_{0}^{2}
$$

The minimization of $\chi_{F}^{2}$ leads to the variation of the identified set of material parameters

$$
\{\delta \boldsymbol{p}\}^{(i+1)}=\left(\left(\left[\boldsymbol{S}_{F}\right]^{(i)}\right)^{t}\left[\boldsymbol{S}_{F}\right]^{(i)}\right)^{-1}\left[\boldsymbol{S}_{F}\right]^{(i)}\left\{\boldsymbol{F}_{m}-\boldsymbol{F}_{c}^{(i)}\right\}
$$


where

$$
\left[\boldsymbol{S}_{F}\right]^{(i)}=\frac{\partial\left\{\boldsymbol{F}_{c}^{(i)}\right\}}{\partial\{\boldsymbol{p}\}}
$$

are the reaction force sensitivities.

Using the same analogy between the algorithmic solution to the problem and the small perturbation computation of the effect of noise, the covariance matrix of the identified parameters with respect to the reaction forces reads

$$
\left[\boldsymbol{C}_{p}^{F}\right]=\left[\boldsymbol{H}_{F}\right]^{-1}
$$

where $\left[\boldsymbol{H}_{F}\right]=\left[\boldsymbol{S}_{F}\right]^{t}\left[\boldsymbol{C}_{F}\right]^{-1}\left[\boldsymbol{S}_{F}\right]=\gamma_{F}^{-2}\left[\boldsymbol{S}_{F}\right]^{t}\left[\boldsymbol{S}_{F}\right]$ is the reaction force Hessian.

The identification based on the combined displacement fields and reaction forces is achieved by minimizing the global functional $\chi_{U F}^{2}[38,37$

$$
\chi_{U F}^{2}=\frac{N_{U}}{N_{U}+N_{F}} \chi_{U}^{2}+\frac{N_{F}}{N_{U}+N_{F}} \chi_{F}^{2}
$$

where the displacement and reaction force functionals have been introduced in Equations 2.11) and 2.17) respectively. The reason for the specific weight of the two functionals originates from a Bayesian foundation for using a weighted quadratic difference including noise covariance. The underlying hypothesis is that the noise is Gaussian and remains so at all stages of its manifestation. The quadratic form is in fact the argument of the exponential in this Gaussian probability distribution. The additivity of the functionals is the counterpart of the statistical independence of the two measurements (i.e., load and images), which implies that probabilities are to be multiplied. The chosen normalization by the noise amplitude in all functionals $\chi$ considered up to now guarantees that 
no additional prefactors are to be considered here. Consistently, if only noise is present (i.e., no model error), the expectation value of $\chi_{U F}^{2}$ amounts to unity.

The minimization leads to the iterative computation of the parameter increment $\{\delta \boldsymbol{p}\}^{(i)}$

$\{\delta \boldsymbol{p}\}^{(i+1)}=\left[\boldsymbol{H}_{U F}\right]^{-1}\left(\frac{1}{2 \gamma_{f}^{2}}\left[\boldsymbol{S}_{U}\right][\boldsymbol{M}]\left(\left\{\boldsymbol{u}_{m}\right\}-\left\{\boldsymbol{u}_{c}\right\}^{(i)}\right)+\frac{1}{\gamma_{F}^{2}}\left[\boldsymbol{S}_{F}\right]\left(\left\{\boldsymbol{F}_{m}\right\}-\left\{\boldsymbol{F}_{c}\right\}^{(i)}\right)\right)$

where the global (i.e., kinematic and static) Hessian is considered $\left[\boldsymbol{H}_{U F}\right]=$

$\left(\left[\boldsymbol{H}_{U}\right]+\left[\boldsymbol{H}_{F}\right]\right)$. The covariance matrix of the identified parameters becomes

$$
\left[\boldsymbol{C}_{\boldsymbol{p}}^{\boldsymbol{U} \boldsymbol{F}}\right]=\langle\{\delta \boldsymbol{p}\} \otimes\{\delta \boldsymbol{p}\}\rangle=\left[\boldsymbol{H}_{U F}\right]^{-1}
$$

The above sections have underlined the similarity of principles at play for extracting information with the least uncertainty from noisy data, both for DIC moving from images to displacement fields, and FEMU going from displacement fields (and load measurements) to material parameters. In this two-step process, one may note that displacement data are only an intermediate quantity, whose computation may imply constraints (e.g., coarse mesh for DIC to be wellconditioned) that are not ideally suited to FEMU. It is thus desirable to merge these two processes into one. This is known as Integrated-DIC, or IDIC, and is now presented.

\subsubsection{Integrated DIC (IDIC)}

Integrated digital image correlation (IDIC) is a global DIC technique that relies on a mechanical choice for the measured displacement field. It allows the user to perform the measurement and identification of the sought parameters in 
only one step. The displacement field is approximated as

$$
\boldsymbol{u}(\boldsymbol{x}, t,\{\boldsymbol{p}\})=\boldsymbol{u}\left(\boldsymbol{x}, t,\{\boldsymbol{p}\}^{(i)}\right)+\left[\frac{\partial \boldsymbol{u}}{\partial\{\boldsymbol{p}\}^{(i)}}\left(\boldsymbol{x}, t,\{\boldsymbol{p}\}^{(i)}\right)\right]\{\delta \boldsymbol{p}\}
$$

at iteration $i$, and the reaction forces

$$
\left\{\boldsymbol{F}_{c}\right\}(t,\{\boldsymbol{p}\})=\left\{\boldsymbol{F}_{c}\right\}\left(t,\{\boldsymbol{p}\}^{(i)}\right)+\left[\frac{\partial\left\{\boldsymbol{F}_{c}\right\}}{\partial\{\boldsymbol{p}\}^{(i)}}\left(t,\{\boldsymbol{p}\}^{(i)}\right)\right]\{\delta \boldsymbol{p}\}
$$

where the corrections $\{\delta \boldsymbol{p}\}$ to the sought parameters become the unknowns 37.

In theory, if the same mesh is used in DIC and for the computation of the sensitivity fields, there should be no difference between a two-step determination and an integrated procedure [38. However, this is valid only for small amplitudes of noise and identical meshes. There are many cases where one can resort to a very fine mesh for the mechanical computation that would not be acceptable for DIC. This very fine mesh is not a problem with respect to IDIC as only a few search directions are chosen, and hence the modeling error due to a coarse mesh can be avoided.

If the same mesh is used and the DIC matrix has been computed as $[\boldsymbol{M}]$, and residuals $\{\boldsymbol{b}\}$, IDIC simply consists of projecting the nodal displacement field onto the sensitivity fields. The Hessian becomes

$$
[\boldsymbol{M}]_{I D I C}=[\boldsymbol{S}]^{t}[\boldsymbol{M}][\boldsymbol{S}]
$$

and hence the amplitudes that can still be read as the increments in the constitutive parameters assume the following expression

$$
\{\delta \boldsymbol{p}\}^{(i)}=[\boldsymbol{M}]_{I D I C}^{-1}[\boldsymbol{S}]^{t}\{\boldsymbol{b}\}
$$


In the following, artificial (i.e., computed) cases will be dealt with and hence one may question the added value of using IDIC. In fact it will mostly allow us to check that intermediate ill-conditioning does not interfere with the effect of noise to prevent convergence on the one hand, and it will lead to a realistic treatment of noise irrespective of its value. The level of noise indicated in Table 1 has been chosen as representative of actual experimental conditions.

Table 1: Displacement and load resolutions, and other characteristic features 37 .

\begin{tabular}{|l|c|c|c|c|c|}
\hline Quantity & $\rho_{0}$ & $\rho_{1}$ & $\gamma_{f}$ & $G_{f}=\left\langle(\nabla f)^{2}\right\rangle^{1 / 2}$ & $a$ \\
\hline Value & $2.5 \mathrm{~N}$ & $4 \times 10^{-4}$ & 233 gray levels & 3800 gray levels/pixel & $13.5 \mu \mathrm{m}$ \\
\hline
\end{tabular}

\section{Elastic law with different parameterizations}

For the identification involving multiple parameters, it is somewhat subjective to choose a given criterion to express the quality of identification by a single number to be optimized. It is desirable that all parameters be determined accurately. However when increasing the quality of one determination degrades that of another one, expressing a preference is difficult. Because of this freedom, one cannot pretend to provide a universal answer. The choice made herein is to focus on the "worst" determination, namely, the one that has the largest uncertainty due to noise, and the optimization is designed to reduce this uncertainty to its minimum. However, it is to be noted that even such a choice is fragile. 
A nonlinear transformation of parameters may lead to a different optimization criterion and hence a different answer.

It is important to note that changing parameters is simple and the above formalism may show that this change can be done before or after the identification, and get the very same result. The difficulty rather lies in the formulation of a relevant criterion. In many cases, an easy way out of this debate is to focus on a specific application (e.g., a part with a given shape subjected to prescribed loads and for which one is interested in a deflection at a particular location) and for which one could reformulate an uncertainty criterion suited to the targeted application, which has no chance of coinciding with any other a priori choice.

The chosen objective is from now on to get the smallest level of the largest eigenvalue of the parameter covariance matrix. Equivalently, the largest level of the smallest eigenvalue of the Hessian is sought. Let us introduce the notation of $\left\{\lambda_{U}\right\}$ for the eigenvalues of the Hessian $\left[\boldsymbol{H}_{U}\right]$, and similarly $\left\{\lambda_{F}\right\},\left\{\lambda_{U F}\right\}$ for $\left[\boldsymbol{H}_{F}\right]$ and $\left[\boldsymbol{H}_{U F}\right]$ respectively.

The choice of the constitutive law parameterization is important [42]. Two parameterizations will be considered in isotropic elasticity. One writing is based on the shear, $\mu$, and bulk, $K$, moduli. The stress-strain relationship reads

$$
\boldsymbol{\sigma}=2 \mu\left(\boldsymbol{\epsilon}-\frac{1}{3} \operatorname{tr}(\boldsymbol{\epsilon}) \boldsymbol{I}\right)+K \operatorname{tr}(\boldsymbol{\epsilon}) \boldsymbol{I}
$$

where $\boldsymbol{\sigma}$ and $\boldsymbol{\epsilon}$ are the stress and strain tensors, respectively, and $\boldsymbol{I}$ the identity tensor. Lamé coefficients are related to Young's modulus $E$ and Poisson's ratio 
$\nu$ by

$$
E=\frac{9 K \mu}{3 K+\mu} \quad \text { and } \quad \nu=\frac{3 K-2 \mu}{2(3 K+\mu)}
$$

The values of the parameters in the example discussed in the following section are given in Table 2

Table 2: Elastic parameters representative of 17-7 PH steel

\begin{tabular}{|l||c|c||c|c|}
\hline State & $E(\mathrm{GPa})$ & $\nu(-)$ & $K(\mathrm{GPa})$ & $\mu(\mathrm{GPa})$ \\
\hline Reference $\left(p_{0}\right)$ & 200 & 0.3 & 166.7 & 76.9 \\
\hline Sensitivity analysis $(p)$ & 204 & 0.306 & 175.3 & 78.5 \\
\hline
\end{tabular}

Two optimizations will be studied. Because of the requirement to use a dimensionless set of parameters, the first chosen parameterization is based on $\left\{\log \left(E / E_{0}\right), \log \left(\nu / \nu_{0}\right)\right\}$ collectively denoted $\{\boldsymbol{p}\}$, and the second on $\left\{\log \left(K / K_{0}\right), \log \left(\mu / \mu_{0}\right)\right\}$ denoted $\{\boldsymbol{q}\}$. Here $E_{0}, \nu_{0}, K_{0}$ and $\mu_{0}$ are nominal values of the chosen parameters. The choice of using a log scale for the moduli is to favor relative uncertainties. Within this configuration the local tangent map between the two bases reads

$$
\left\{\begin{array}{c}
\frac{\mathrm{d} K}{K} \\
\frac{\mathrm{d} \mu}{\mu}
\end{array}\right\}=\left[\begin{array}{cc}
1 & \frac{2 \nu}{(1-2 \nu)} \\
1 & \frac{-\nu}{(1+\nu)}
\end{array}\right]\left\{\begin{array}{c}
\frac{d E}{E} \\
\frac{d \nu}{\nu}
\end{array}\right\} \equiv[\boldsymbol{A}]\left\{\begin{array}{c}
\frac{d E}{E} \\
\frac{d \nu}{\nu}
\end{array}\right\}
$$

where $[\boldsymbol{A}]$ is the change of basis matrix. About a current point, the increments $\{\mathrm{d} \boldsymbol{q}\}$ and $\{\mathrm{d} \boldsymbol{p}\}$ are linearly related by the above $[\boldsymbol{A}]$ linear transformation. Figure 3 shows the incremental vectors $\{\mathrm{d} \boldsymbol{q}\}$ in the $\{\mathrm{d} \boldsymbol{p}\}$ plane. It is to be emphasized that these vectors are rotated and scaled differently so that the $\{\boldsymbol{p}\} \rightarrow\{\boldsymbol{q}\}$ 
mapping is not unitary (i.e., norm of vectors will not be preserved). The consequences of this simple observation are now studied regarding the covariance matrices.

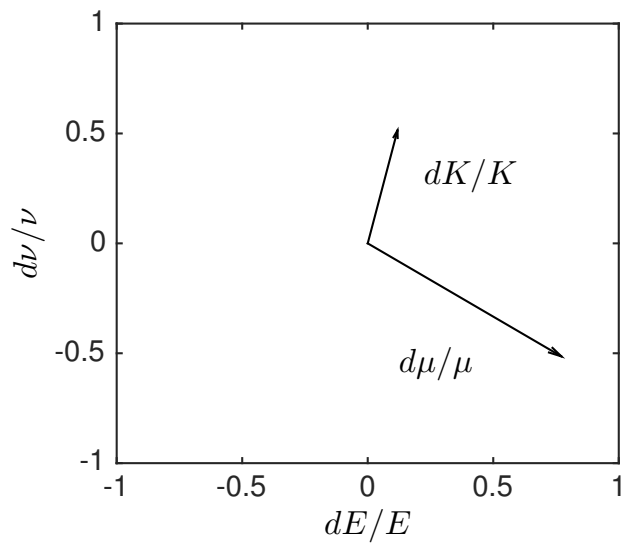

Figure 3: Local map of the $\{\log (K), \log (\mu)\}$ parameterization in the $\{\log (E), \log (\nu)\}$ basis

The covariance matrix in the first set of coordinates $\{\boldsymbol{p}\}$ may be diagonalized as

$$
\left[\boldsymbol{C}_{\boldsymbol{p}}\right]=\langle\{\delta \boldsymbol{p}\} \otimes\{\delta \boldsymbol{p}\}\rangle=[\boldsymbol{V}]^{t}[\boldsymbol{D}][\boldsymbol{V}]
$$

where $[\boldsymbol{V}]$ is the matrix gathering the eigenvectors and $[\boldsymbol{D}]$ the diagonal matrix of eigenvalues. In the second basis, the covariance matrix $\left[\boldsymbol{C}_{\boldsymbol{q}}\right]$ reads

$$
\left[\boldsymbol{C}_{\boldsymbol{q}}\right]=\langle\{\delta \boldsymbol{q}\} \otimes\{\delta \boldsymbol{q}\}\rangle=[\boldsymbol{A}]^{t}[\boldsymbol{V}]^{t}[\boldsymbol{D}][\boldsymbol{V}][\boldsymbol{A}]
$$

A singular value decomposition of $[\boldsymbol{A}]$ leads to the relationship $[\boldsymbol{A}]=\left[\boldsymbol{U}_{1}\right][\boldsymbol{S}]\left[\boldsymbol{U}_{2}\right]$ where $\left[\boldsymbol{U}_{1}\right],\left[\boldsymbol{U}_{2}\right]$ are unitary, and $[\boldsymbol{S}]$ symmetric and positive-definite. Thus the 
covariance matrix becomes

$$
\begin{aligned}
{\left[\boldsymbol{C}_{\boldsymbol{q}}\right] } & =\left[\boldsymbol{U}_{2}\right]^{t}[\boldsymbol{S}]\left[\boldsymbol{U}_{1}\right]^{t}[\boldsymbol{V}]^{t}[\boldsymbol{D}][\boldsymbol{V}]\left[\boldsymbol{U}_{1}\right][\boldsymbol{S}]\left[\boldsymbol{U}_{2}\right] \\
& =\left[\boldsymbol{U}_{2}\right]^{t}[\boldsymbol{S}]\left[\boldsymbol{W}_{\mathbf{1}}\right]^{t}[\boldsymbol{D}]\left[\boldsymbol{W}_{\mathbf{1}}\right][\boldsymbol{S}]\left[\boldsymbol{U}_{2}\right]
\end{aligned}
$$

where $\left[\boldsymbol{W}_{\mathbf{1}}\right]$ is also unitary. In the present case, the matrix $[\boldsymbol{A}]$ is equal to

$$
[\boldsymbol{A}]=\left[\begin{array}{cc}
1 & 1.5 \\
1 & -0.23
\end{array}\right]
$$

and its singular decomposition leads to the three matrices

$$
\left[\boldsymbol{U}_{1}\right]=\left[\begin{array}{cc}
-0.96 & -0.27 \\
-0.27 & 0.96
\end{array}\right] \quad[\boldsymbol{S}]=\left[\begin{array}{cc}
1.85 & 0 \\
0 & 0.93
\end{array}\right] \quad\left[\boldsymbol{U}_{2}\right]=\left[\begin{array}{cc}
-0.66 & 0.75 \\
-0.75 & -0.66
\end{array}\right]
$$

The criterion was chosen earlier as being the largest eigenvalue of the covariance matrix. It is to be stressed that a change of basis will not preserve such a criterion (it would if $[\boldsymbol{A}]$ were unitary). The largest eigenvalue of $\left[\boldsymbol{C}_{\boldsymbol{q}}\right]$ is less than or equal to the largest eigenvalue of $\left[\boldsymbol{C}_{\boldsymbol{p}}\right]$ times the square of the largest eigenvalue of $[\boldsymbol{S}]$ (i.e., this is only a bound). If a criterion based on the uncertainty "volume" $\operatorname{det}\left(\left[\boldsymbol{C}_{\boldsymbol{p}}\right]\right)$ were chosen, then a deterministic relationship could have been derived

$$
\operatorname{det}\left(\left[\boldsymbol{C}_{\boldsymbol{q}}\right]\right)=\operatorname{det}\left(\left[\boldsymbol{C}_{\boldsymbol{p}}\right]\right) \operatorname{det}([\boldsymbol{S}])^{2}
$$

suggesting a more favorable look to represent the results in the basis corresponding to the $\{\boldsymbol{p}\}$ parameterization rather than the $\{\boldsymbol{q}\}$ one, since $\operatorname{det}([\boldsymbol{S}])^{2} \approx 3$.

This example shows that although one can freely move from one representation to another one without loss of information, the choice of a specific form 
criterion and a fortiori of its quantitative value may convey different appreciations of the quality of the identification. It is not suggested here to use a specific form of criterion, but simply to underline its consequences. In other terms, if the user desires to focus on one or several parameters, a suitable parameterization should be chosen. Therefore the corresponding experimental and identification procedures will result from the above considerations.

\section{Geometry optimization}

The following section now reports on the shape optimization of the cruciform sample whose geometry is shown in Figure 1 and designed for biaxial experiments. Furthermore, the triangular loading path presented in Figure 2 is applied. The constitutive law of the specimen is assumed to be elastic (see Section 4.1).

\subsection{Analysis of an elastic case}

The IDIC procedure is discussed assuming a linear isotropic elastic constitutive law based on the $\{\boldsymbol{p}\}$ set of parameters, namely, $p_{1}=\log \left(E / E_{0}\right)$ and $p_{2}=\log \left(\nu / \nu_{0}\right)$ where $E_{0}$ and $\nu_{0}$ are chosen as representative of 17-7 PH stainless steel [39, 37] and listed in Table 2. The sensitivity fields are first discussed. They are obtained in all the sequel from a numerical finite difference based on a $2 \%$ variation of the parameters (Table 2).

First, let us note that the Young's modulus sensitivity field $\left[\boldsymbol{S}_{U}\right]_{E}$ is equal to zero in that case. This is in accordance with the fact that Dirichlet boundary conditions are prescribed and no load information is used. A change in Young's 
modulus would only affect the load but not the displacement field for such a linear behavior. In contrast, the sensitivity fields related to the Poisson's ratio $\left[\boldsymbol{S}_{U}\right]_{\nu}$ whose horizontal component is shown in Figure 4 is nontrivial. This sensitivity field is computed for a geometry such that $r=2 \mathrm{~mm}$ and at the maximum amplitude loading with equi-biaxial tension (point $B$ of the loading path of Figure 21. Due to the fact that the thickness is uniform over the whole sample, the sensitivity is low close to the center of the sample, but interestingly it varies more strongly across the vertical arm of the specimen (i.e., close to uniaxial tension).

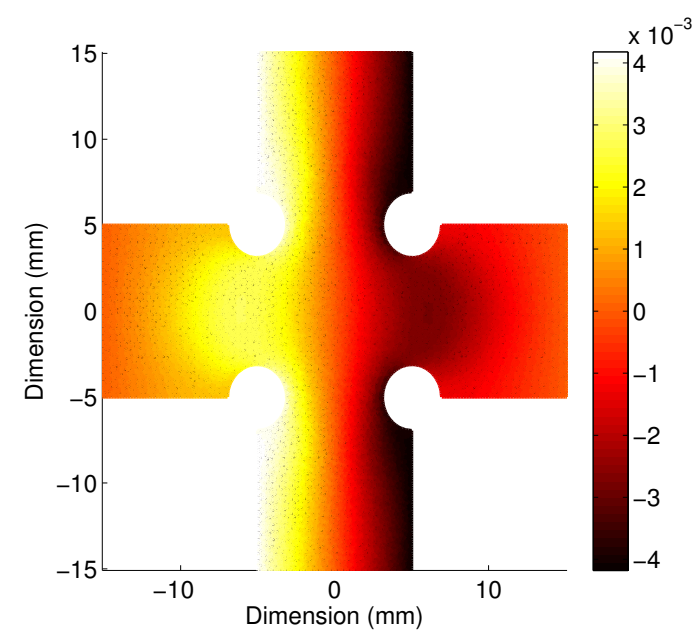

Figure 4: Sensitivity field $\left[\boldsymbol{S}_{U}\right]_{\nu} \cdot \boldsymbol{e}_{\mathbf{1}}$ expressed in $\mathrm{mm}$ in the parameterization $\{\boldsymbol{p}\}$ related to Poisson's ratio

Using the second parameterization $\{\boldsymbol{q}\}=\left\{\log \left(K / K_{0}\right), \log \left(\mu / \mu_{0}\right)\right\}$, Figure 5 shows the horizontal component of the sensitivity fields $\left[\boldsymbol{S}_{U}\right]_{K}$ and $\left[\boldsymbol{S}_{U}\right]_{\mu}$ at point $B$. These fields are both non zero, as changing $K$ at fixed $\mu$ or $\mu$ at 
fixed $K$ does alter the Poisson's ratio. However, as only one degree of freedom matters, it is expected that $\left[\boldsymbol{S}_{U}\right]_{K},\left[\boldsymbol{S}_{U}\right]_{\mu}$ and $\left[\boldsymbol{S}_{U}\right]_{\nu}$ are all collinear

$$
\begin{gathered}
{\left[\boldsymbol{S}_{U}\right]_{K}=a\left[\boldsymbol{S}_{U}\right]_{\nu}} \\
{\left[\boldsymbol{S}_{U}\right]_{\mu}=b\left[\boldsymbol{S}_{U}\right]_{\nu}}
\end{gathered}
$$

where $a$ and $b$ can be evaluated from the expression of matrix $[\boldsymbol{A}]$ (see Equation (3.3) ) as $a=\left(A^{-1}\right)_{21} \approx 0.58$ and $b=\left(A^{-1}\right)_{22} \approx-0.58$.

Figure 6 shows the change of the only nonzero eigenvalue of the instantaneous Hessian along the loading path. Furthermore, this graph contains parabolas, of which two originate from $\mathrm{O}(\mathrm{OA}$ and $\mathrm{BO})$. This means that these two radial parts bring the same qualitative information, with an eigen value of the Hessian that scales as the square of the displacement amplitude (hence the parabolas). Along the $\mathrm{AB}$ path, the sensitivity fields do not preserve a fixed orientation through time, there is no simple relationship between instantaneous values and those of the radial parts of the load path, even though a single degree of freedom is sensitive.

Let us stress that this section illustrates the notion of sensitivity field through instantaneous loading stages. However, when performing an actual identification, the Hessian to be considered is the summation over time of the instantaneous Hessians, i.e., over the entire loading path. Similarly right hand side members are to be summed over time.

Figures 6 and 7 show the level of the (nonzero) eigenvalues of instantaneous Hessians. It would be an exact property if the noise variance were constant, which is not true for the load uncertainty, but the deviation is almost negligible. 


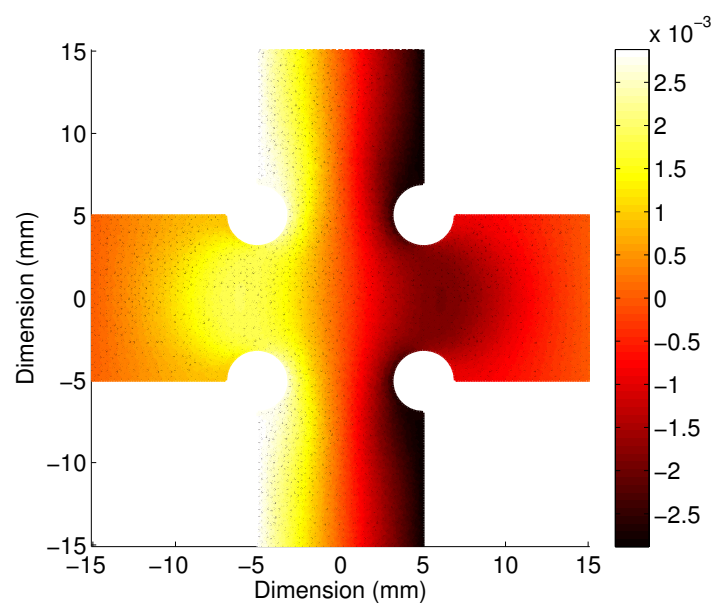

(a)

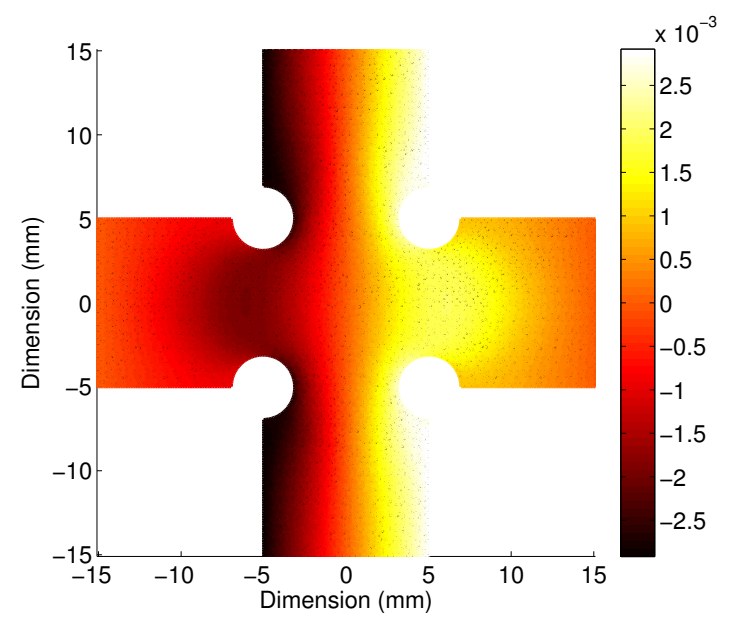

(b)

Figure 5: (a) Horizontal component of the sensitivity field $\left[\boldsymbol{S}_{U}\right]_{K}$ relative to the bulk modulus and (b) of the sensitivity field $\left[\boldsymbol{S}_{U}\right]_{\mu}$ relative to the shear modulus at loading point $B$. The displacements are expressed in $\mathrm{mm}$ 


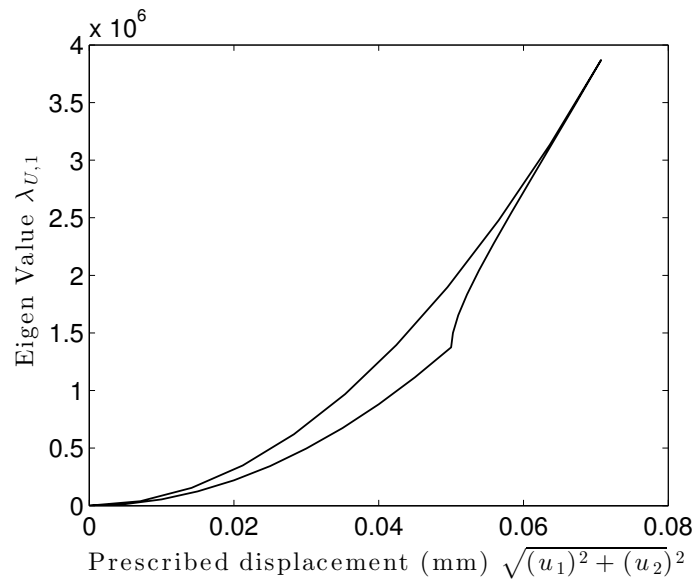

Figure 6: eigenvalue $\lambda_{U, 1}$ of the Hessian $\left[\boldsymbol{H}_{U}\right]$ with the $\left\{\log \left(E / E_{0}\right), \log \left(\nu / \nu_{0}\right)\right\}$ parameterization

The kinematic Hessian $\left[\boldsymbol{H}_{U}\right]$ is evaluated at each step of the loading path. Figure 6 shows only the nonzero eigenvalue $\lambda_{U, 1}$ of $\left[\boldsymbol{H}_{U}\right]$. The maximum level of $\lambda_{U, 1}$ is reached for point $B$, but the behavior is different for the loading and unloading parts. The load dissymmetry reduces the value of $\lambda_{U, 1}$. For an equivalent amplitude $\sqrt{u_{1}^{2}+u_{2}^{2}}$ the eigenvalue is lower for the asymmetric history.

Figure 7 (a) shows the largest eigenvalue $\lambda_{F, 1}$ related to $\left[\boldsymbol{H}_{F}\right]$. The maximum level of $\lambda_{F, 1}$ is again reached for point $B$. However, a different behavior is observed for the second eigenvalue, $\lambda_{F, 2}$ (Figure $7(\mathrm{~b})$ ). While the maximum level of the largest eigenvalue is reached for the maximum amplitude, the maximum level for $\lambda_{F, 2}$ is reached for point $A$, i.e., for the most asymmetric boundary condition. When the loads are equal in all branches of the sample, the second eigenvalue vanishes. Therefore, the equibiaxial loading history (in terms of loads 
$F$ ) only provides information for the dominant eigenvalue (whose eigenvector is essentially aligned with $\log (E)$ ). Furthermore, the different behaviors of the two eigenvalues originate from the two distinct material parameters. Last, let us stress that the ratio between the two eigenvalues related to $\left[\boldsymbol{H}_{F}\right]$ is in the range of a few hundreds. This important contrast leads to severe noise sensitivity issues when performing the inversion based on the static data only.

\subsection{Geometry optimization in elasticity}

Geometry optimization is now considered. Let us recall that the least uncertainty is sought, or with the criterion discussed in Section 3 , the smallest eigenvalue of the Hessian should be maximized. In order to be consistent it is important to ensure that the yield stress is not exceeded along the loading path. However, the highest stress depends critically on the geometry, namely, the smaller the fillet radius $r$ the higher the stress concentration. Hence it is decided to adjust the maximum displacement $d^{*}$ of the sample arms so that the maximum von Mises equivalent stress matches a lower bound on the yield stress, chosen to be $\sigma_{y}=1300 \mathrm{MPa}$, which is representative of 17-7 PH stainless steel in TH 1050 condition [39.

The eigenvalues of the kinematic, $\left[\boldsymbol{H}_{U}\right]$, and static, $\left[\boldsymbol{H}_{F}\right]$, Hessians, which are integrated over the whole loading history, for each studied geometry are displayed in Figure 8 As earlier noted in the purely kinematic case the Young's modulus cannot be determined, and hence $\lambda_{U, 2}=0$ (since $\left[\boldsymbol{H}_{U}\right]_{E E}=\left[\boldsymbol{H}_{U}\right]_{E \nu}=$ $\left.\left[\boldsymbol{H}_{U}\right]_{\nu E}=0\right)$. The other eigenvalue, $\lambda_{U, 1}$, has a rather smooth variation with an optimal value at about $r=r^{o p t} \approx 1.7 \mathrm{~mm}$ for which the uncertainty on the 


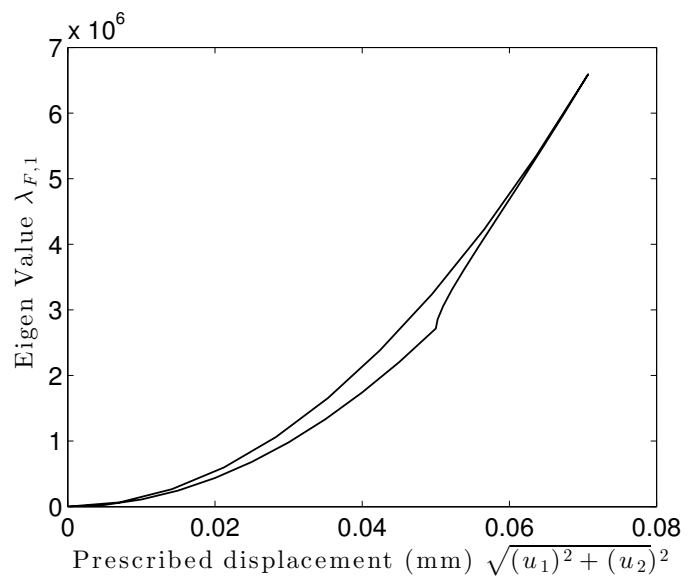

(a)

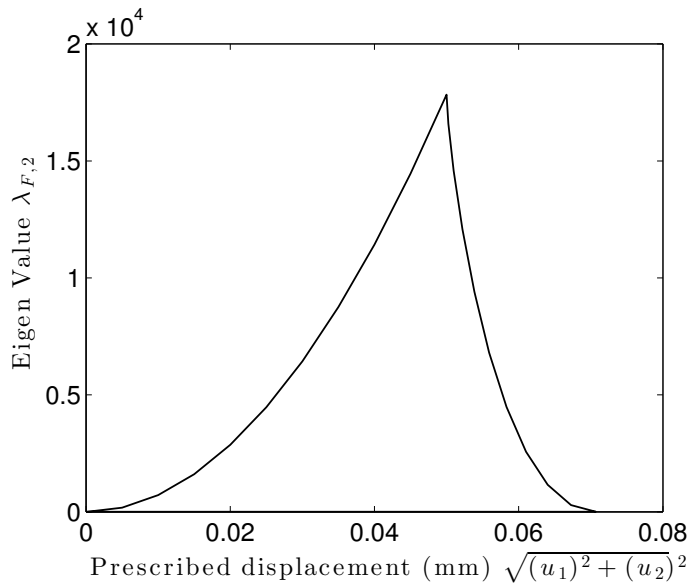

(b)

Figure 7: Largest eigenvalue $\lambda_{F, 1}$ (a) and smallest one $\lambda_{F, 2}$ (b) of the Hessian $\left[\boldsymbol{H}_{F}\right]$ with the $\left\{\log \left(E / E_{0}\right), \log \left(\nu / \nu_{0}\right)\right\}$ parameterization

Poisson's ratio is minimal.

Considering the reaction forces, Figure 8(b) shows the eigenvalues of the Hessian $\left[\boldsymbol{H}_{F}\right]$ with the fillet radius $r$. Both eigenvalues $\lambda_{F, 1}$ and $\lambda_{F, 2}$ display 


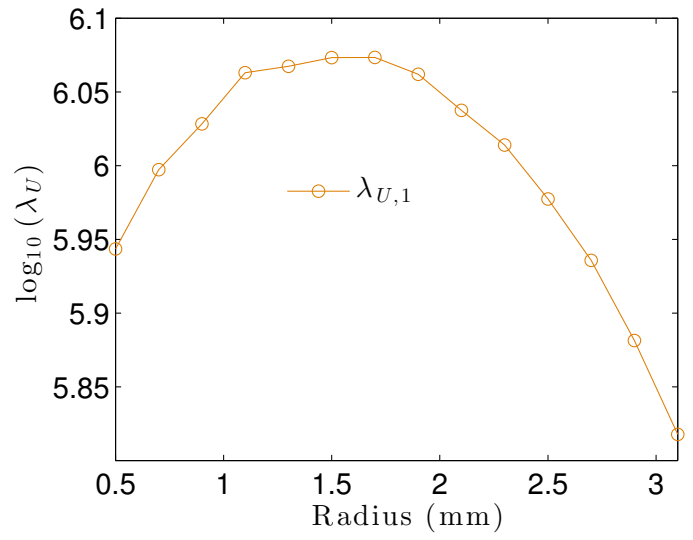

(a)

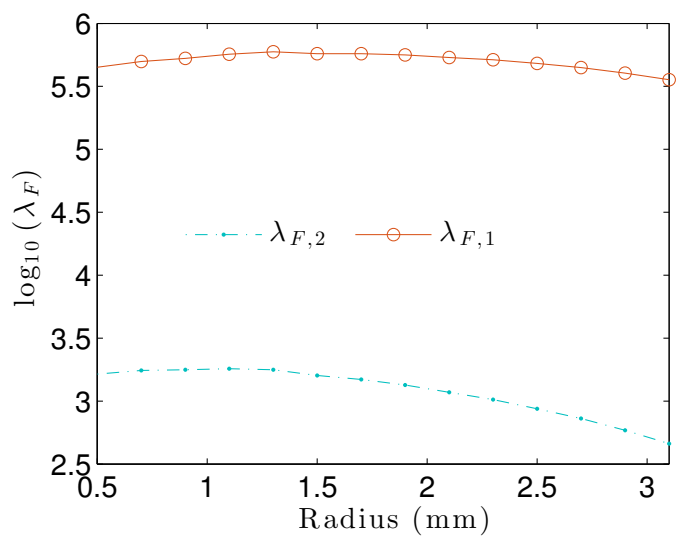

(b)

Figure 8: Eigenvalue $\lambda_{U, 1}$ of the Hessian $\left[\boldsymbol{H}_{U}\right]$ and $\lambda_{F, 1}$ and $\lambda_{F, 2}$ of the Hessian $\left[\boldsymbol{H}_{F}\right]$ in linear and isotropic elasticity for different fillet radii with the $\left\{\log \left(E / E_{0}\right), \log \left(\nu / \nu_{0}\right)\right\}$ parameterization

a similar behavior as that of $\lambda_{U, 1}$ with a smooth maximum in the considered range. However, the maximum value of each eigenvalue is reached for a different radius, $r_{1}^{o p t}=1.7 \mathrm{~mm}$ for $\lambda_{F, 1}$ and $r_{2}^{o p t}=1.4 \mathrm{~mm}$ for $\lambda_{F, 2}$. If only reaction 
forces were considered in the identification, the criterion based on the largest value of the smallest eigenvalue would lead to a fillet radius $r_{2}^{\text {opt }}$.

It is worth noting that the largest eigenvalue assumes a comparable value for the displacement-based and the load-based identification, stating that no obvious hierarchy is expected when using kinematic or static information. Rather they should complement each other. Moreover, the influence of the loading history is not studied herein. However, it is worth noting that the latter would affect the eigenvalues, and thus, the value of the optimized fillet radius.

For a different parameterization $\left\{\log \left(K / K_{0}\right), \log \left(\mu / \mu_{0}\right)\right\}$, Figure 9 shows the eigenvalues of the two Hessians $\left[\boldsymbol{H}_{U}\right]$ and $\left[\boldsymbol{H}_{F}\right]$. As discussed in Section 3 the second eigenvalue of $\left[\boldsymbol{H}_{U}\right]$ is null. Since a single eigenvalue remains, the optimal value of the fillet radius has to be identical to that observed with the $\{\boldsymbol{p}\}$ parametrization when dealing with $\left[\boldsymbol{H}_{U}\right]$. Considering the reaction forces, the behavior of the two eigenvalues is very close to that observed in Figure 8b. Some slight changes of at most a factor of 4 could be anticipated due to the singular value decomposition of matrix $[\boldsymbol{A}]$.

Figure 10 (a) shows the eigenvalues of the global Hessian $\left[\boldsymbol{H}_{U F}\right]$ using the $\left\{\log \left(E / E_{0}\right), \log \left(\nu / \nu_{0}\right)\right\}$ parameterization. The largest eigenvalue remains in the same range as previously observed with $\left[\boldsymbol{H}_{U}\right]$ and $\left[\boldsymbol{H}_{F}\right], \lambda_{1} \approx 10^{6}$. However, the most noticeable change lies in the second eigenvalue. From 0 with $\left[\boldsymbol{H}_{U}\right]$, and $10^{3}$ with $\left[\boldsymbol{H}_{F}\right]$ it now reaches about $3 \times 10^{5}$ when both static and kinematic data are combined at their best. This rise shows that these two sources of information are very complementary, and, schematically, if the load allows one to evaluate 


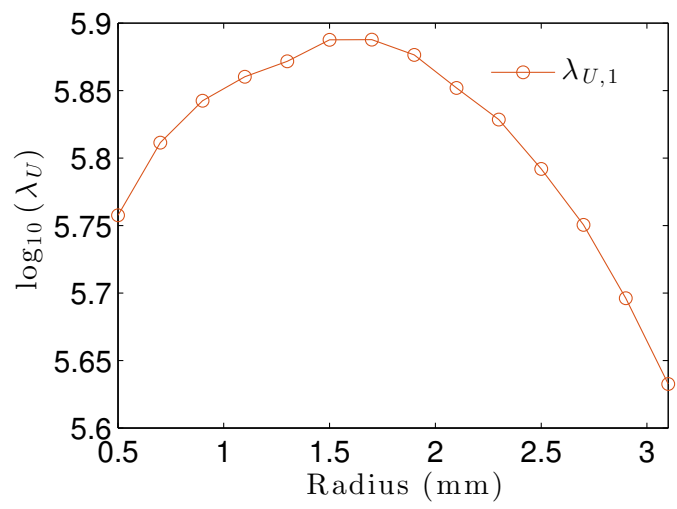

(a)

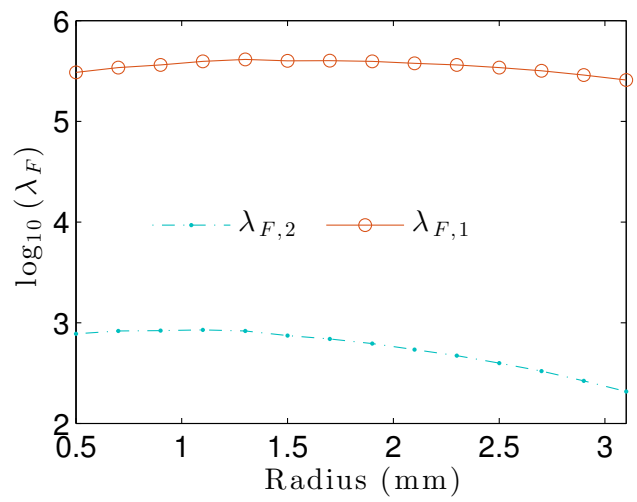

(b)

Figure 9: Eigenvalue $\lambda_{U, 1}$ of the Hessian $\left[\boldsymbol{H}_{U}\right]$ and $\lambda_{F, 1}$ and $\lambda_{F, 2}$ of the Hessian $\left[\boldsymbol{H}_{F}\right]$ in linear and isotropic elasticity for different fillet radii with the $\left\{\log \left(K / K_{0}\right), \log \left(\mu / \mu_{0}\right)\right\}$ parameterization. The second eigenvalue $\lambda_{U, 2}$ is equal to zero

the Young's modulus, the DIC analysis captures the Poisson's ratio influence. The optimal fillet radius is again observed to be of the order $r^{o p t}=1.7 \pm 0.4 \mathrm{~mm}$. 
Last, the influence of the parameterization can again be considered. Figure 10(b) is the equivalent for the $\{\boldsymbol{p}\}$ parameters of Figure 10.(a) for $\{\boldsymbol{q}\}$. The comparison of both sub-figures shows that the $\left\{\log \left(E / E_{0}\right), \log \left(\nu / \nu_{0}\right)\right\}$ parameterization is more favorable since the lowest eigenvalue is higher compared with that obtained with $\left\{\log \left(K / K_{0}\right), \log \left(\mu / \mu_{0}\right)\right\}$ as could have been expected from the singular value decomposition of $[\boldsymbol{A}]$. This change is however marginal and simply shows the care with which the optimization criterion should be formulated. Fortunately, this change in the values of $\lambda$ does not affect much the optimal radius $r$, which remains of the order of $r^{\text {opt }}=1.7 \pm 0.4 \mathrm{~mm}$.

The above geometry optimization investigated a rather simple class with only one degree of freedom (i.e., one fillet radius). The same principles can be applied to a larger class of variants. Practical restrictions may come from the computational cost of the study. The following subsection aims to illustrate an optimization procedure that allows a wider range of geometry variations to be considered at a modest additional cost.

\subsection{Geometry optimization in elasticity with different fillets}

The section aims to investigate the influence of the four fillet radii and to propose a strategy to optimize their sizes. First, with all the fillet radii being equal an optimal value is obtained as shown in Section 4.2. Second, the investigation is performed by only modifying three fillet radii with the same value and seeking the new optimal value while keeping one filet radius equal to the previous optimal value. Then, the second fillet radii is also fixed with the new optimum value. This process is repeated until all the optimum fillet radii 


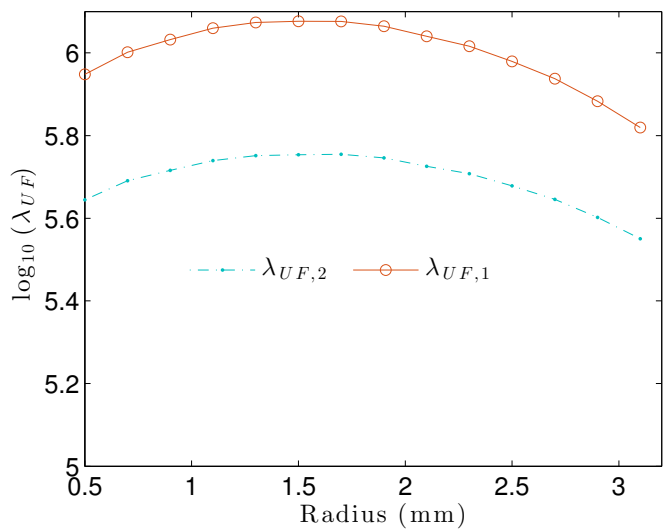

(a) $\left\{\log \left(E / E_{0}\right), \log \left(\nu / \nu_{0}\right)\right\}$ parameterization

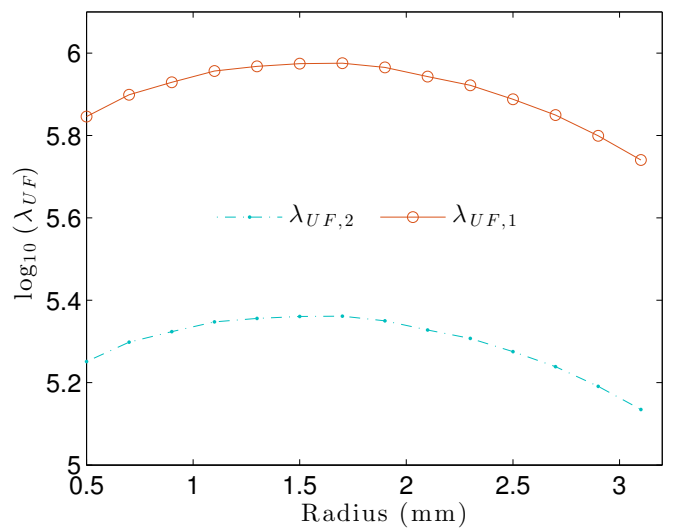

(b) $\left\{\log \left(K / K_{0}\right), \log \left(\mu / \mu_{0}\right)\right\}$ parameterization

Figure 10: Eigenvalues $\lambda_{U F}$ of Hessian $\left[\boldsymbol{H}_{U F}\right]$ with two different parameterizations

are found. The fillet radii are optimized in the trigonometric order from the top left corner. The same loading history is considered (Figure 2).

Figure 11 shows the eigenvalues $\lambda_{U F}$ of Hessian $\left[\boldsymbol{H}_{U F}\right]$ for the four optimiza- 
tion steps and for the $\left\{\log \left(E / E_{0}\right), \log \left(\nu / \nu_{0}\right)\right\}$ parameterization. The second optimization step does not strongly change the results and because less fillet radii are modified, the changes of the eigenvalues are less significant. Consequently, four identical fillet radii equal to $r=1.7 \pm 0.4 \mathrm{~mm}$ correspond to the optimal shape for the identification of elastic parameters.

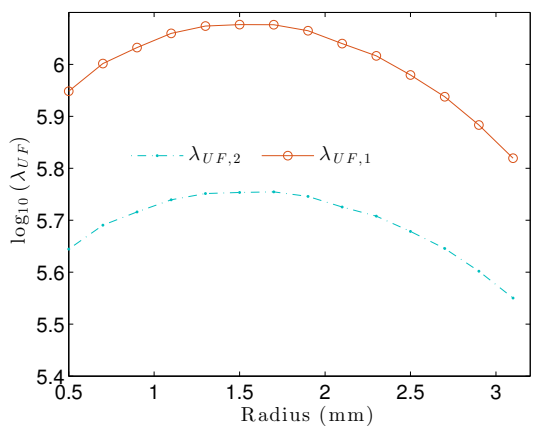

(a) four fillet radii variation

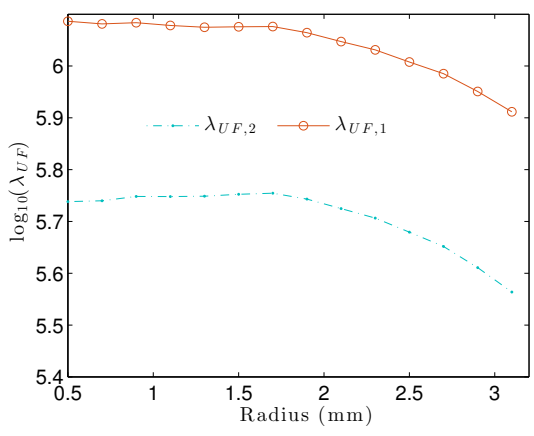

(c) two fillet radii variation

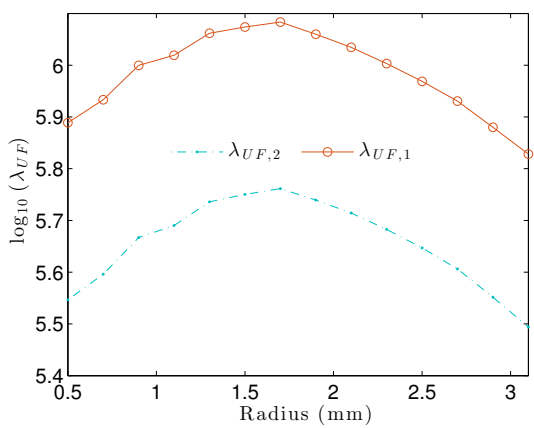

(b) three fillet radii variation

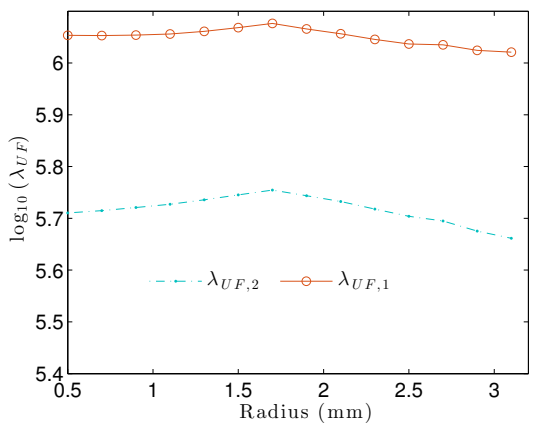

(d) one fillet radius variation

Figure 11: Eigenvalues $\lambda_{U F}$ of Hessian $\left[\boldsymbol{H}_{U F}\right]$ for the four optimization steps

\subsection{Geometry optimization in elastoplasticity}

Geometry optimization has been addressed up to now within the framework 
of linear elasticity. It has been emphasized that the entire optimization procedure as presented herein is transparent to the complexity of the parameters used in the modeling (although the cost of computing the sensitivity fields will be directly dependent on the sophistication of the chosen constitutive law). This statement also emphasizes that the "optimality" of a specimen geometry will never be universally optimal but only within a given framework. In this section, the initial geometry optimization (above shown to lead to an at least local optimum for a larger class of geometries) with a single degree of freedom (i.e., four identical fillet radii) is reconsidered for an elastoplastic material. The chosen law is a standard one with linear kinematic hardening [46]

$$
\dot{\boldsymbol{X}}=\frac{2}{3} C \dot{\boldsymbol{\epsilon}}_{p l}
$$

where $C$ is a material parameter, $\dot{\boldsymbol{\epsilon}}_{p l}$ the plastic strain rate tensor, and $\boldsymbol{X}$ the back-stress. The yield surface $J_{2}(\boldsymbol{\sigma}-\boldsymbol{X})=\sigma_{0}$ is defined such that $J_{2}$ is von Mises' equivalent stress and $\sigma_{0}$ the yield stress. The maximum amplitude prescribed displacement $d^{*}$ is defined such that the maximum equivalent plastic strain is equal to $10 \%$. The prescribed loading history is shown in Figure 2 The parameterization is identical to that chosen for the elastic case, namely, $\{q\}=\left\{\log \left(E / E_{0}\right), \log \left(\nu / \nu_{0}\right), \log \left(\sigma / \sigma_{0}\right), \log \left(C / C_{0}\right)\right\}$. Table 3 gathers the reference values of the material parameters.

Figure 12 shows the eigenvalues of $\left[\boldsymbol{H}_{U F}\right]$ when the four fillet radii are equal. The two smallest eigenvalues have similar trends and values as the linear elastic case. The maximum level of the smallest eigenvalue $\lambda_{U F, 4}$ is reached when $r=$ $1.5 \pm 0.3 \mathrm{~mm}$. This value is slightly less than that observed in elasticity. Although 
Table 3: Value of the material parameters

\begin{tabular}{|l|c|c|c|c|}
\hline Parameter & $E$ & $\nu$ & $\sigma_{0}$ & $C$ \\
\hline Value & $200 \mathrm{GPa}$ & 0.3 & $1300 \mathrm{MPa}$ & $10 \mathrm{GPa}$ \\
\hline
\end{tabular}

in the present case the optimal geometries for elasticity and elastoplasticity are quite close, it is worth emphasizing that different constitutive laws do not necessarily lead to the same optimal configuration.

Further, the fact that four material parameters are now investigated does not significantly alter the values of the smallest eigenvalues of the Hessian, even if the latter is slightly smaller than the linear elastic case at the initial step of the optimization. At the end of the process their values are almost equal $\left(\log _{10}\left(\lambda_{\text {min }}^{\text {elas }}\right) \approx \log _{10}\left(\lambda_{\text {min }}^{\text {plas }}\right) \approx 5.8\right)$. This result indicates that the elastic parameters are likely to be more difficult to identify than the hardening parameters. Consequently, an optimization only based on elastic parameters is already giving a good indication for the optimization. Its additional advantage lies in the fact that the simulations are much faster than for more complex constitutive equations.

Conversely, several orders of magnitude are observed between the four eigenvalues, which are significantly higher than in the elastic analysis (Figure 10. This observation shows that the hardening parameters are not as sensitive to noise as the elastic ones, which is to be expected since the strain levels (as displacement fluctuations) are more important in plasticity than in elasticity. 


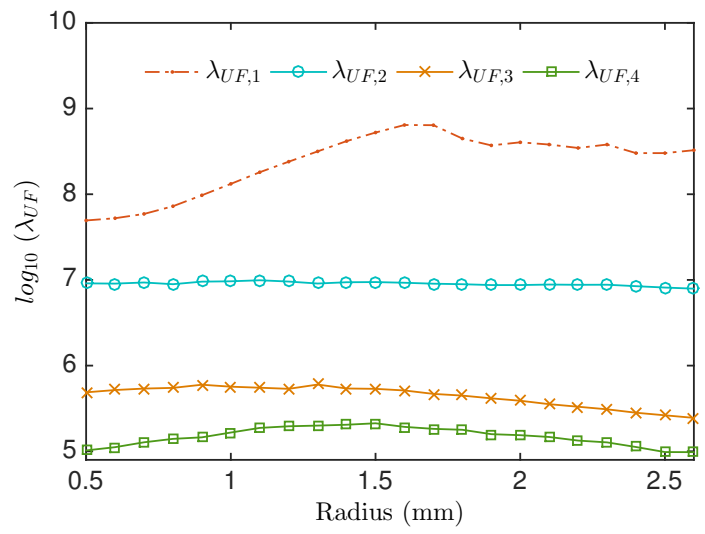

Figure 12: Eigenvalues $\lambda_{U F}$ of Hessian $\left[\boldsymbol{H}_{U F}\right]$ for an elastoplastic law with linear kinematic hardening when all the fillet radii are varying

\subsection{Geometry optimization in elastoplasticity with different fillets}

Figure 13 (a) shows the eigenvalues of $\left[\boldsymbol{H}_{U F}\right]$ when one fillet radius is kept constant and equal to $r=1.5 \mathrm{~mm}$ (i.e., the optimal case for four equal sizes). The other three fillet radii have the same value. The lowest eigenvalue becomes greater than what was achieved in the previous case. The optimal value is $r \approx 2.6 \mathrm{~mm}$ for which $\lambda_{U F, 3}$ and $\lambda_{U F, 4}$ are identical. Last, it is noteworthy that several local minima are encountered. This originates from the elastoplastic kinematic behavior that influences the sensitivities that are no longer trivial and linear.

Last, Figure 13(b) shows the eigenvalues of $\left[\boldsymbol{H}_{U F}\right]$ when three fillet radii are kept constant. The maximum level of $\lambda_{U F, 4}$ is achieved for a fillet radius equal to $r=2.6 \mathrm{~mm}$ (at the intersection of $\lambda_{U F, 4}$ and $\lambda_{U F, 3}$ ). Furthermore, another maximum is reached for $r=0.8 \mathrm{~mm}$. Contrary to elasticity, the present analysis 
shows that having various fillet radii is more favorable than having equal ones.

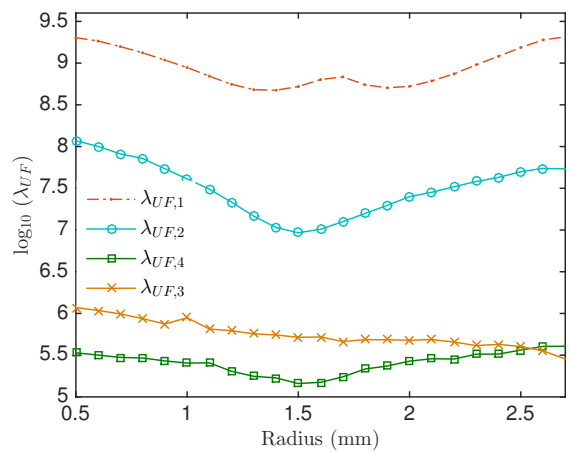

(a) one fillet radius variation

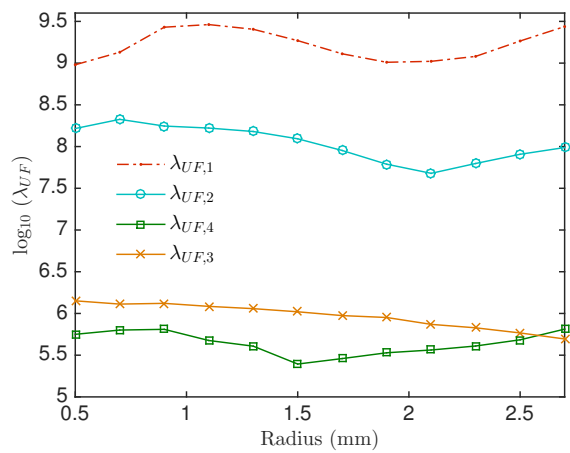

(b) three fillet radii variation

Figure 13: Eigenvalues $\lambda_{U F}$ of Hessian $\left[\boldsymbol{H}_{U F}\right]$ for the optimization steps 2 and 3

This result can be understood by analyzing the sensitivity fields. Figure 14 shows the sensitivity fields $\left[\boldsymbol{S}_{U}\right]_{p} \cdot \boldsymbol{e}_{\mathbf{1}}$ for the four material parameters at loading point $B$. The sensitivity fields $\left[\boldsymbol{S}_{U}\right]_{\sigma_{0}}$ and $\left[\boldsymbol{S}_{U}\right]_{C}$ are not vanishing in the center of the specimen. This would occur in a fully symmetric geometry. In terms of levels, the two sensitivity fields are several orders of magnitude higher in plasticity in comparison with elasticity as was already observed when analyzing the eigenvalues of $\left[\boldsymbol{H}_{U F}\right]$ with four identical fillet radii (Figure 12 . 


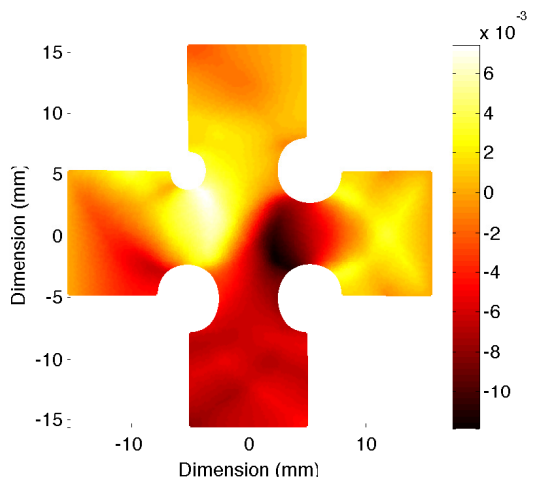

(a)

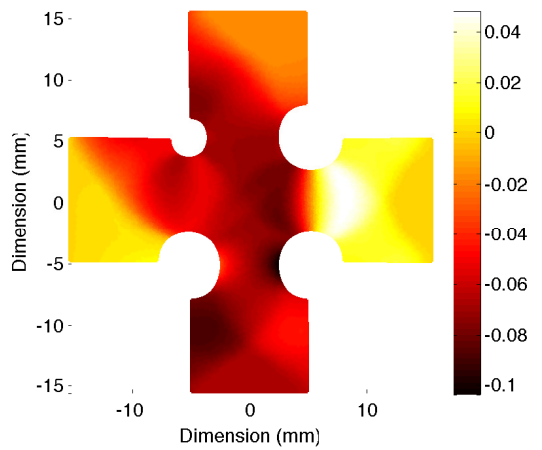

(c)

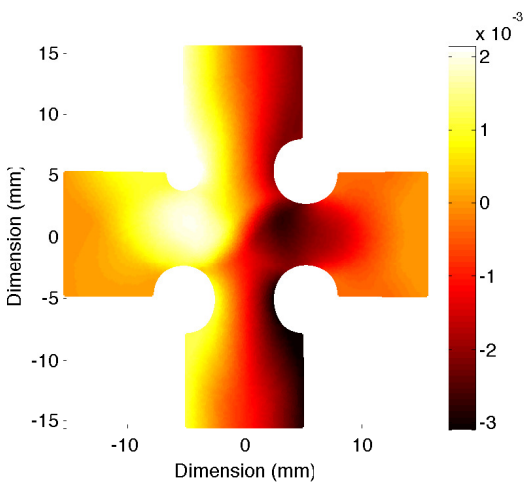

(b)

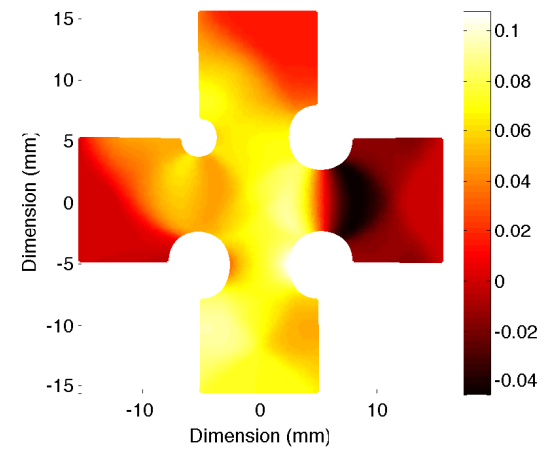

(d)

Figure 14: Sensitivity fields $\left[\boldsymbol{S}_{U}\right]_{E} \cdot \boldsymbol{e}_{\mathbf{1}}(\mathrm{a}),\left[\boldsymbol{S}_{U}\right]_{\nu} \cdot \boldsymbol{e}_{\mathbf{1}}(\mathrm{b}),\left[\boldsymbol{S}_{U}\right]_{\sigma_{0}} \cdot \boldsymbol{e}_{\mathbf{1}}(\mathrm{c})$, and $\left[\boldsymbol{S}_{U}\right]_{C} \cdot \boldsymbol{e}_{\mathbf{1}}(\mathrm{d})$ at point $B$. The displacements are expressed in $\mathrm{mm}$ 


\section{Conclusion}

A complete identification procedure has been described, which starts from the acquisition of experimental data (i.e., load levels and images) to the extraction of material parameters whereby uncertainties could be tracked all the way down to the identification step. It treats FEMU and DIC on the same footing and embraces both steps into a single one via IDIC. With this procedure in hand, the cruciform specimen geometry of a biaxial test could be optimized in such a way that the final uncertainty on the identified constitutive parameters is minimized.

The proposed procedure fully exploits the wealth of information provided by full-field measurements, and weights this information according to its value, namely, its inverse uncertainty. As emphasized in the introduction, the advent of full-field measurements allows to aim for the optimization directly to the final goal, and not to intermediate conditions (such as homogeneity) that were necessary for the exploitation of the test, as needed when strain gauges were to be used.

The optimization is based on the minimization of the worst uncertainty although different criteria may have been chosen. The present approach considers the entire metrological chain (i.e., from the random pattern of DIC to finite element simulations for the computation of sensitivity fields) to account for the measurement resolution. As a result, the optimization is dedicated to a specific specimen and even more to its expected behavior. Different results are found for the two investigated laws. The optimization in linear elasticity leads to four 
identical fillet radii whereas the elastoplastic optimization leads to various fillet radii. However, the maximum level of the minimum eigenvalue is not significantly different between elastic and elastoplastic optimizations. The advantage of the former is that the computation time is significantly lower and provides a good first estimate (i.e., 3 hours in comparison with 10-hour computation time on a PC with an 8-core Intel Xeon E5 processor). The result would presumably be different if the study would focus on a specific material parameter.

In the treated example, it is to be observed that sensitive regions are favored with respect to those where the strain field is the most uniform when the four fillet radii are identical. Such a marked difference implies that the here-derived optimized shape is significantly different from those obtained without resorting to full-field measurements. In particular the arms of the cruciform sample and the areas surrounding the fillet exhibit a greater sensitivity to the constitutive parameters than the center and hence are to be exploited. Conversely, when the radii are not identical, the central part of the sample is also contributing to the elastoplastic parameter sensitivity, which globally increases, thereby decreasing the overall sensitivity to noise.

Finally, the loading path has been considered here together with the specimen geometry in order to ensure the relevance of elasticity or a maximum strain level in plasticity. A more elaborate optimization of the loading path could be envisioned within the same goal of reducing the identification uncertainty, which will be investigated in future studies by following the same optimization path as proposed herein. 


\section{Acknowledgments}

It is a pleasure to acknowledge the support of Région Ile de France (DICCIT and THERMOFLUIDE-RT projects). 


\section{References}

[1] R. Hill, A theory of the yielding and plastic flow of anisotropic metals, Proceedings of the Royal Society of London. Series A, Mathematical and Physical Sciences 193 (1033) (1948) 281-297. doi:10.1098/rspa.1948. 0045 .

[2] I. Wilson, D. J. White, Cruciform specimens for biaxial fatigue tests: An investigation using finite-element analysis and photoelastic-coating techniques, The Journal of Strain Analysis for Engineering Design 6 (1) (1971) 27-37. doi:10.1243/03093247V061027.

[3] M. W. Brown, K. J. Miller, A theory for fatigue failure under multiaxial stress-strain conditions, Proceedings of the Institution of Mechanical Engineers 187 (1) (1973) 745-755. doi:10.1243/PIME_PROC_1973_187_161_02.

[4] D. A. Kelly, Problems in creep testing under biaxial stress systems, Journal of strain analysis for engineering design 11 (1) (1976) 1-6. doi:10.1243/ $03093247 V 111001$.

[5] G. Ferron, A. Makinde, Design and development of a biaxial strenght testing device, Journal of Testing and Evaluation 16 (1988) 253-256. doi $: 10.1520 /$ JTE10375J

[6] A. Makinde, Mise au point d'un dispositif original de traction biaxial: application à l'étude expérimentale de l'écrouissage des métaux sous différents chemins de déformation., Ph.D. thesis, Université de Poitiers (1986). 
[7] A. Makinde, L. Thibodeau, K. Neale, Development of an apparatus for biaxial testing using cruciform specimens, Experimental Mechanics 32 (2) (1992) 138-144. doi:10.1007/BF02324725

URL http://dx.doi.org/10.1007/BF02324725

[8] T. Kuwabara, S. Ikeda, K. Kuroda, Measurement and analysis of differential work hardening in cold-rolled steel sheet under biaxial tension, Journal of Materials Processing Technology $80 a ̂ 81$ (1998) 517 - 523. doi:http://dx.doi.org/10.1016/S0924-0136(98)00155-1. URL http://www.sciencedirect.com/science/article/pii/ S0924013698001551

[9] P. Boisse, A. Gasser, G. Hivet, Analyses of fabric tensile behaviour: determination of the biaxial tension-strain surfaces and their use in forming simulations, Composites Part A: Applied Science and Manufacturing 32 (10) (2001) 1395-1414. doi:10.1016/S1359-835X(01)00039-2.

[10] N. Muzyka, Equipment for testing sheet structural materials under biaxial tension. part 2. testing by biaxial loading in the plane of the sheet, Strength of Materials 34 (2) (2002) 206-212. doi:10.1023/A:1015326913484. URL http://dx.doi.org/10.1023/A\%3A1015326913484

[11] M. Teaca, I. Charpentier, M. Martiny, G. Ferron, Identification of sheet metal plastic anisotropy using heterogeneous biaxial tensile tests, International Journal of Mechanical Sciences 52 (4) (2010) 572 - 580. doi:http://dx.doi.org/10.1016/j.ijmecsci.2009.12.003. 
URL

http://www.sciencedirect.com/science/article/pii/

S0020740309002616

[12] D. E. Green, Etude expérimentale et numérique du comportement biaxial des tôles minces, Ph.D. thesis, Department of the Faculty of Engineering of the Université de Sherbrooke (janvier 1996).

[13] D. Lecompte, A. Smits, H. Sol, J. Vantomme, D. V. Hemelrijck, Mixed numerical-experimental technique for orthotropic parameter identification using biaxial tensile tests on cruciform specimens, International Journal of Solids and Structures 44 (5) (2007) 1643 - 1656. doi:http://dx.doi.org/10.1016/j.ijsolstr.2006.06.050.

URL http://www.sciencedirect.com/science/article/pii/ S0020768306002848

[14] T. Kuwabara, Advances in experiments on metal sheets and tubes in support of constitutive modeling and forming simulations, International Journal of Plasticity 23 (3) (2007) 385 - 419. doi:http://dx.doi.org/10.1016/j.ijplas.2006.06.003

URL http://www.sciencedirect.com/science/article/pii/ S0749641906001124

[15] T. Kuwabara, Advances of plasticity experiments on metal sheets and tubes and their applications to constitutive modeling, AIP Conference Proceedings 778 (1) (2005) 20-39. doi:http://dx.doi.org/10.1063/1.2011190 
URL http://scitation.aip.org/content/aip/proceeding/aipcp/10.

$1063 / 1.2011190$

[16] J. Eftis, D. Jones, H. Liebowitz, Load biaxiality and fracture: Synthesis and summary, Engineering Fracture Mechanics 36 (4) (1990) 537 - 574. doi:http://dx.doi.org/10.1016/0013-7944(90)90112-T.

URL http://www.sciencedirect.com/science/article/pii/ $001379449090112 \mathrm{~T}$

[17] A. Hannon, P. Tiernan, A review of planar biaxial tensile test systems for sheet metal, Journal of Materials Processing Technology 198 (1-3) (2008)

1 - 13. doi:http://dx.doi.org/10.1016/j.jmatprotec.2007.10.015

URL http://www.sciencedirect.com/science/article/pii/ S0924013607009533

[18] A. Chen, F. Matthews, A review of multiaxial/biaxial loading tests for composite materials, Composites 24 (5) (1993) 395 - 406. doi:http://dx.doi.org/10.1016/0010-4361(93)90247-6.

URL http://www.sciencedirect.com/science/article/pii/ 0010436193902476

[19] J. Boehler, S. Demmerle, S. Koss, A new direct biaxial testing machine for anisotropic materials, Experimental Mechanics 34 (1) (1994) 1-9. doi: $10.1007 / \mathrm{BF} 02328435$

URL http://dx.doi .org/10.1007/BF02328435

[20] M. Johlitz, S. Diebels, Characterisation of a polymer using biaxial tension 
tests. part I: Hyperelasticity, Archive of Applied Mechanics 81 (10) (2011)

1333-1349. doi:10.1007/s00419-010-0480-1.

URL http://dx.doi.org/10.1007/s00419-010-0480-1

[21] M. Merklein, M. Biasutti, Development of a biaxial tensile machine for characterization of sheet metals, Journal of Materials Processing Technology 213 (6) (2013) 939 - 946. doi:http: //dx.doi.org/10.1016/j.jmatprotec.2012.12.005

URL http://wWW.sciencedirect.com/science/article/pii/ S0924013612003676

[22] E. Hoferlin, A. V. Bael, P. V. Houtte, G. Steyaert, C. D. MarÃ@e, Biaxial tests on cruciform specimens for the validation of crystallographic yield loci, Journal of Materials Processing Technology 80 â81 (1998) 545 - 550. doi:http://dx.doi.org/10.1016/S0924-0136(98)00123-X. URL http://www.sciencedirect.com/science/article/pii/ S092401369800123X

[23] J. F. Wilson, B. L. Kinsey, Y. P. Korkolis, Development of a biaxial loading frame for sheet metal, Journal of Manufacturing Processes 15 (4) (2013) 580 - 585. doi:http://dx.doi.org/10.1016/j.jmapro.2013.06.008.

URL http://www.sciencedirect.com/science/article/pii/ S1526612513000777

[24] A. Makinde, L. Thibodeau, K. Neale, D. Lefebvre, Design of a biaxial extensometer for measuring strains in cruciform specimens, Experimental 
Mechanics 32 (2) (1992) 132-137. doi:10.1007/BF02324724.

URL http://dx.doi.org/10.1007/BF02324724

[25] S. Demmerle, J. Boehler, Optimal design of biaxial tensile cruciform specimens, Journal of the Mechanics and Physics of Solids 41 (1) (1993) 143 - 181. doi:http://dx.doi.org/10.1016/0022-5096(93)90067-P.

URL http://www.sciencedirect.com/science/article/pii/ $002250969390067 \mathrm{P}$

[26] Y. Yu, M. Wan, X.-D. Wu, X.-B. Zhou, Design of a cruciform biaxial tensile specimen for limit strain analysis by $\{\mathrm{FEM}\}$, Journal of Materials Processing Technology 123 (1) (2002) $67 \quad-70$. doi:http://dx.doi.org/10.1016/S0924-0136(02)00062-6.

URL http://www.sciencedirect.com/science/article/pii/ S0924013602000626

[27] J.-Y. Cognard, V. Feuardent, J.-M. Virely, Optimisation of a structure for biaxial mechanical tests, Integrated Design and Manufacturing in Mechanical Engineering (1997) 495-504doi:10.1007/978-94-011-5588-5_50. URL http://dx.doi.org/10.1007/978-94-011-5588-5_50

[28] Y. Hanabusa, H. Takizawa, T. Kuwabara, Numerical verification of a biaxial tensile test method using a cruciform specimen, Journal of Materials Processing Technology 213 (6) (2013) 961 - 970. doi:http://dx.doi.org/10.1016/j.jmatprotec.2012.12.007. 
URL

http://www.sciencedirect.com/science/article/pii/

S0924013612003780

[29] C. Doudard, Détermination rapide des propriétés en fatigue à grand nombre de cycles à partir d'essais d'échauffement, Ph.D. thesis, Ecole Normale Supérieure de Cachan (2004).

[30] E. Lamkanfi, W. V. Paepegem, J. Degrieck, C. Ramault, A. Makris, D. V. Hemelrijck, Strain distribution in cruciform specimens subjected to biaxial loading conditions. part 2: Influence of geometrical discontinuities, Polymer Testing 29 (1) (2010) $132 \quad-138$. doi:http://dx.doi.org/10.1016/j.polymertesting.2009.10.002.

URL http://www.sciencedirect.com/science/article/pii/ S0142941809001731

[31] K. T. Kavanagh, R. W. Clough, Finite element applications in the characterization of elastic solids, International Journal of Solids and Structures 7 (1) (1971) 11 - 23. doi:http://dx.doi.org/10.1016/0020-7683(71) $90015-1$

URL http://www.sciencedirect.com/science/article/pii/ 0020768371900151

[32] S. Avril, M. Bonnet, A.-S. Bretelle, M. Grédiac, F. Hild, P. Ienny, F. Latourte, D. Lemosse, S. Pagano, E. Pagnacco, F. Pierron, Overview of identification methods of mechanical parameters based on full-field measurements, Experimental Mechanics 48 (4) (2008) 381-402. doi:10.1007/ 
s11340-008-9148-y

URL http://dx.doi .org/10.1007/s11340-008-9148-y

[33] M. Grédiac, F. Hild, Full-Field Measurements and Identification in Solid Mechanics, ISTE, Wiley, 2012.

URL http: //books . google.fr/books?id=3bNmDdp5POkC

[34] S. Schmaltz, K. Willner, Comparison of different biaxial tests for the inverse identification of sheet steel material parameters, Strain 50 (5) (2014) 389403. doi:10.1111/str.12080.

URL http://dx.doi.org/10.1111/str.12080

[35] H. Leclerc, J. Périé, S. Roux, F. Hild, Integrated digital image correlation for the identification of mechanical properties, Vol. LNCS 5496, Springer, Berlin, 2009, pp. 161-171.

[36] J. Réthoré, A fully integrated noise robust strategy for the identification of constitutive laws from digital images, Int. J. Num. Meth. Eng. 84 (6) (2010) 631-660.

[37] M. Bertin, F. Hild, S. Roux, F. Mathieu, H. Leclerc, P. Aimedieu, Integrated digital image correlation applied to elasto-plastic constitutive laws in an optimized biaxial experiment, Journal of Strain Analysis for Engineering Designdoi:10.1177/0309324715614759.

[38] F. Mathieu, H. Leclerc, F. Hild, S. Roux, Estimation of elastoplastic parameters via weighted femu and integrated DIC, Experimental Mechanics 
55 (1) (2015) 105-119. doi:10.1007/s11340-014-9888-9.

URL http://dx.doi.org/10.1007/s11340-014-9888-9

[39] AK-Steel, Mechanical characteristics 17-7 PH steel, AK-Steel (2013).

[40] A. Leybold-Herbert, Axial-load fatigue tests on $17-7 \mathrm{ph}$ stainless steel under constant-amplitude loading, NASA TN D-439 62 71013, National Aeronautics and Space Administration, Langley Research Center Langley Field, Va. (October 1960).

[41] Y. Tick-Hon, Cyclic plasticity of 17-7 precipitation-hardenable semiaustenic stainless steel, Ph.D. thesis, University of Toronto (1998).

[42] A. Tarantola, Inverse Problems Theory. Methods for Data Fitting and Model Parameter Estimation, Elsevier Applied Science, Southampton (UK), 1987.

[43] F. Hild, S. Roux, Comparison of local and global approaches to digital image correlation, Experimental Mechanics 52 (9) (2012) 1503-1519. doi: $10.1007 / \mathrm{s} 11340-012-9603-7$

URL http://dx.doi.org/10.1007/s11340-012-9603-7

[44] G. Besnard, F. Hild, S. Roux, Finite-element" displacement fields analysis from digital images: Application to portevin-le châtelier bands, Experimental Mechanics 46 (6) (2006) 789-803. doi:10.1007/ s11340-006-9824-8.

URL http://dx.doi.org/10.1007/s11340-006-9824-8 
[45] R. Gras, H. Leclerc, F. Hild, S. Roux, J. Schneider, Identification of a set of macroscopic elastic parameters in a 3d woven composite: Uncertainty analysis and regularization, International Journal of Solids and Structures (2014) DOI 10.1016/j.ijsolstr.2013.12.023doi: 10.1016/j.ijsolstr.2013.12.023.

URL http://www.sciencedirect.com/science/article/pii/ S0020768313004964

[46] W. Prager, B. U. D. of Applied Mathematics, U. S. O. of Naval Research, U. S. N. D. B. of Ships, A New Method of Analyzing Stresses and Strains in Work-hardening Plastic Solids, Technical report (Brown University. Division of Applied Mathematics), Division of Applied Mathematics, Brown University, 1955.

URL http://books .google.nl/books?id=UbDntgAACAAJ 


\section{Main notations}

$\begin{array}{ll}\chi_{f} & \text { digital image correlation residual } \\ \gamma_{f} & \text { standard deviation of gray levels } \\ \gamma_{F} & \text { standard resolution of the load measurement } \\ \Omega & \text { region of interest } \\ \boldsymbol{u}(\boldsymbol{x}) & \text { displacement field vector } \\ {[\boldsymbol{M}]} & \text { global correlation matrix } \\ {\left[\boldsymbol{S}_{U}\right]} & \text { displacement sensitivity matrix } \\ {\left[\boldsymbol{S}_{F}\right]} & \text { force sensitivity matrix } \\ {\left[\boldsymbol{C}_{\boldsymbol{U}}\right]} & \text { covariance matrix of measured nodal displacements } \\ {\left[\boldsymbol{C}_{\boldsymbol{F}}\right]} & \text { covariance matrix of measured load } \\ {\left[\boldsymbol{C}_{p}^{\bullet}\right]} & \text { covariance matrix of identified material parameters using a } \\ {\left[\boldsymbol{H}_{U}\right]} & \text { method labeled by } \bullet \\ {\left[\boldsymbol{H}_{F}\right]} & \text { kinematic Hessian } \\ {\left[\boldsymbol{H}_{U F}\right]} & \text { static Hessian }\end{array}$




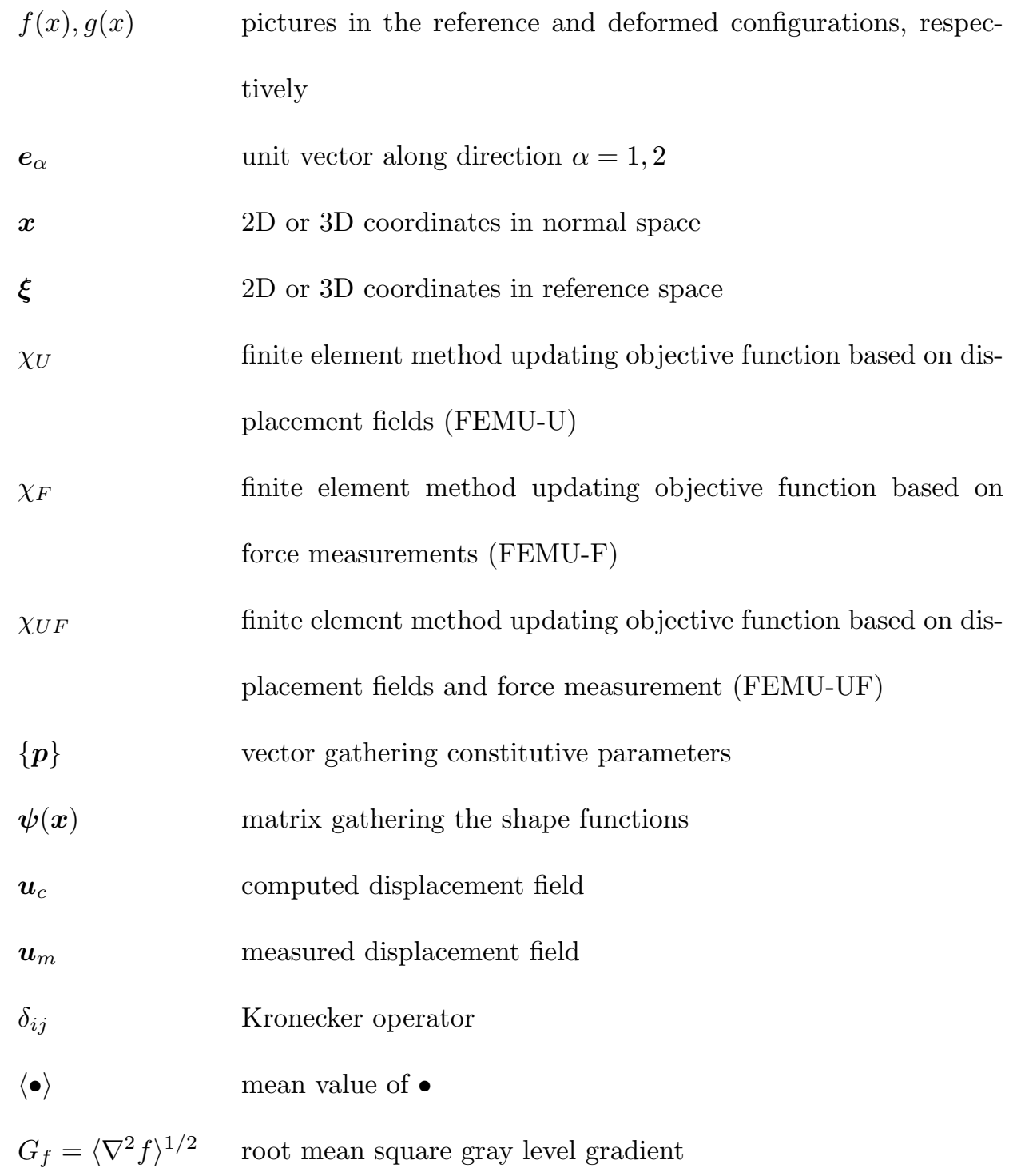




\section{List of Figures}

$1 \quad$ Mesh and geometry of the analyzed specimen with a fillet radius $r=2 \mathrm{~mm} \ldots \ldots \ldots$. . . . . . . . . . . . 8

2 (a) Triangular loading history prescribed in the simulation. The squares depict the computed steps. (b) Boundary conditions prescribed on the sample in terms of longitudinal displacements.

With the chosen loading history the center of the specimen is motionless . . . . . . . . . . . . . . . . . . 10

3 Local map of the $\{\log (K), \log (\mu)\}$ parameterization in the

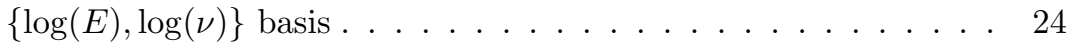

$4 \quad$ Sensitivity field $\left[\boldsymbol{S}_{U}\right]_{\nu} \cdot \boldsymbol{e}_{\boldsymbol{1}}$ expressed in $\mathrm{mm}$ in the parameterization $\{\boldsymbol{p}\}$ related to Poisson's ratio $\ldots . . . . . . . . ~ 27$

5 (a) Horizontal component of the sensitivity field $\left[\boldsymbol{S}_{U}\right]_{K}$ relative to the bulk modulus and (b) of the sensitivity field $\left[\boldsymbol{S}_{U}\right]_{\mu}$ relative to the shear modulus at loading point $B$. The displacements are expressed $\mathrm{in} \mathrm{mm}$. . . . . . . . . . . . . . . . . . 29

6 eigenvalue $\lambda_{U, 1}$ of the Hessian $\left[\boldsymbol{H}_{U}\right]$ with the

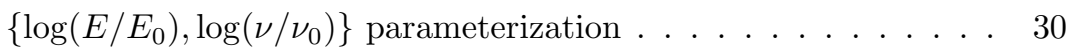

7 Largest eigenvalue $\lambda_{F, 1}(\mathrm{a})$ and smallest one $\lambda_{F, 2}(\mathrm{~b})$ of the Hes$\operatorname{sian}\left[\boldsymbol{H}_{F}\right]$ with the $\left\{\log \left(E / E_{0}\right), \log \left(\nu / \nu_{0}\right)\right\}$ parameterization. . . 32

8 Eigenvalue $\lambda_{U, 1}$ of the Hessian $\left[\boldsymbol{H}_{U}\right]$ and $\lambda_{F, 1}$ and $\lambda_{F, 2}$ of the Hessian $\left[\boldsymbol{H}_{F}\right]$ in linear and isotropic elasticity for different fillet radii with the $\left\{\log \left(E / E_{0}\right), \log \left(\nu / \nu_{0}\right)\right\}$ parameterization . . . . . 33 
9 Eigenvalue $\lambda_{U, 1}$ of the Hessian $\left[\boldsymbol{H}_{U}\right]$ and $\lambda_{F, 1}$ and $\lambda_{F, 2}$ of the Hes$\operatorname{sian}\left[\boldsymbol{H}_{F}\right]$ in linear and isotropic elasticity for different fillet radii with the $\left\{\log \left(K / K_{0}\right), \log \left(\mu / \mu_{0}\right)\right\}$ parameterization. The second eigenvalue $\lambda_{U, 2}$ is equal to zero . . . . . . . . . . . . . . . . . 35

10 Eigenvalues $\lambda_{U F}$ of Hessian $\left[\boldsymbol{H}_{U F}\right]$ with two different parameterizations . . . . . . . . . . . . . . . 37

11 Eigenvalues $\lambda_{U F}$ of Hessian $\left[\boldsymbol{H}_{U F}\right]$ for the four optimization steps 38

12 Eigenvalues $\lambda_{U F}$ of Hessian $\left[\boldsymbol{H}_{U F}\right]$ for an elastoplastic law with linear kinematic hardening when all the fillet radii are varying. . 41

13 Eigenvalues $\lambda_{U F}$ of Hessian $\left[\boldsymbol{H}_{U F}\right]$ for the optimization steps 2 and 3 .......................... . . 42

14 Sensitivity fields $\left[\boldsymbol{S}_{U}\right]_{E} \cdot \boldsymbol{e}_{\mathbf{1}}(\mathrm{a}),\left[\boldsymbol{S}_{U}\right]_{\nu} \cdot \boldsymbol{e}_{\mathbf{1}}(\mathrm{b}),\left[\boldsymbol{S}_{U}\right]_{\sigma_{0}} \cdot \boldsymbol{e}_{\mathbf{1}}(\mathrm{c})$, and $\left[\boldsymbol{S}_{U}\right]_{C} \cdot \boldsymbol{e}_{\mathbf{1}}(\mathrm{d})$ at point $B$. The displacements are expressed in $\mathrm{mm} \quad 43$ 